\title{
Thermodynamic and holographic information dual to volume
}

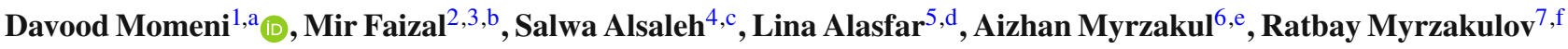 \\ ${ }^{1}$ Department of Physics, College of Science, Sultan Qaboos University, P.O. Box 36, Al-Khodh 123, Muscat, Sultanate of Oman \\ ${ }^{2}$ Irving K. Barber School of Arts and Sciences, University of British Columbia Okanagan, Kelowna, BC V1V 1V7, Canada \\ ${ }^{3}$ Department of Physics and Astronomy, University of Lethbridge, Lethbridge, AB T1K 3M4, Canada \\ ${ }^{4}$ Department of Physics and Astronomy, College of Science King Saud University, Riyadh 11451, Saudi Arabia \\ ${ }^{5}$ Université Clermont Auvergne, 4 Avenue Blaise Pascal, 63710 Aubière, France \\ ${ }^{6}$ Physics Department, Nazarbayev University, Astana, Kazakhstan \\ ${ }^{7}$ Eurasian International Center for Theoretical Physics and Department of General and Theoretical Physics, Eurasian National University, Astana \\ 010008, Kazakhstan
}

Received: 28 December 2017 / Accepted: 10 September 2018 / Published online: 22 September 2018

(C) The Author(s) 2018

\begin{abstract}
In this paper, we will analyze the connection between the fidelity susceptibility, the holographic complexity and the thermodynamic volume. We will regularize the fidelity susceptibility and the holographic complexity by subtracting the contribution of the background AdS spacetime from the deformation of the AdS spacetime. It will be demonstrated that this regularized fidelity susceptibility has the same behavior as the thermodynamic volume and that the regularized complexity has a very different behavior. As the information dual to different volumes in the bulk would be measured by the fidelity susceptibility and the holographic complexity, this paper will establish a connection between thermodynamics and information dual to a volume.
\end{abstract}

\section{Introduction}

It has been observed from various studies done in different branches of physics that the laws of physics are related to the ability of an observer to process relevant information [1,2]. Thus, it seems to indicate that the laws of physics are information theoretical processes. As the informational theoretical process deal with the processing of information, it is important to measure the loss of information during such a process. It is possible to measure this loss of information using the concept of entropy, and so that the entropy is a very important quantity in information theory. However, as the laws of

\footnotetext{
a e-mail: davood@squ.edu.om

b e-mail: mirfaizalmir@googlemail.com

c e-mail: salwams@ksu.edu.sa

de-mail: lina.alasfar@outlook.fr

e e-mail: aizhan.myrzakul@nu.edu.kz

f e-mail: rmyrzakulov@gmail.com
}

physics are also information theoretical processes, entropy is an important physical quantity. In fact, using the Jacobson formalism, it is possible to obtain the geometric structure of spacetime by assuming a certain scaling behavior of the maximum entropy of a region of space [3,4]. This scaling behavior of maximum entropy has been motivated from the holographic principle $[5,6]$. The holographic principle states that the number of degrees of freedom in any region of space is equal to the number of degrees of freedom on the boundary of that region of space. The holographic principle is also the basis of the the AdS/CFT conjecture [7]. The AdS/CFT correspondence states that the supergravity/string theory in the bulk of an AdS spacetime is dual to the superconformal field theory on its boundary. The AdS/CFT correspondence has been used to quantify the concept of entanglement in conformal field theory. This is because the AdS/CFT correspondence can be used to holographically calculate quantum entanglement entropy of a conformal field theory from the bulk AdS spacetime [8-10]. This is done by defining $\gamma_{A}$ as the $(d-1)$-minimal surface extended for a subsystem $A$ with boundary $\partial A$. The holographic entanglement entropy for such a subsystem can be expressed in terms of the gravitational constant $G_{d+1}$ and the area of the minimal surface Area $[A]$ as $[11,12]$

Entropy $_{A}=\frac{\operatorname{Area}[A]\left(\gamma_{A}\right)}{4 G_{d+1}}$.

Since the entropy is related to the loss of information, it can be calculated holographically from the area of a minimal surface. However, it is not only important to understand the loss of information during an information theoretical process, but it is also important to understand the difficulty to process that information during such a process. This can be quanti- 
fied using the concept of complexity. As the laws of physics are informational theoretical processes, it is expected that complexity will become an important physical quantity, and the fundamental laws of physics will be expressed in terms of complexity. It may be noted that complexity has already been used to understand the behavior of certain condensed matter systems [13-15]. It has also been used for analyzing molecular physics $[16,17]$. In fact, even quantum computational systems have been studied using the concept of complexity [18]. The studies done on black hole information indicate that the information might not be lost, but it would be left in such a state that it would not be effectively possible to recover it from that state [19]. This indicates that complexity might be an important quantity that can be used to understand the black hole information paradox. It has been proposed that the complexity can also be holographically calculated from the bulk AdS spacetime [20]. In fact, it has been proposed that the complexity would be dual to a volume $V$ in the bulk AdS spacetime and hence it can be defined as follows [21,22]:

Complexity $=\frac{V}{8 \pi R G_{d+1}}$,

where $R$ is the radius of the curvature. There are various different ways to define a radius in AdS, and so we have different proposals for complexity. It is possible to use the same minimal surface which was used to calculate the holographic entanglement entropy, and define this volume as the volume enclosed by such a surface $V=V(\gamma)$ [23]. However, this quantify diverges, so we will regularize it by subtracting the contribution of a background AdS $V(\gamma)$ Ads from the deformation of AdS $V(\gamma)_{\text {AdS }}$, and define

$\Delta \mathcal{C}=\frac{V(\gamma)_{\mathrm{DAdS}}-V(\gamma)_{\mathrm{AdS}}}{8 \pi R G_{d+1}}$.

This quantity will be finite, and we shall call this quantity as the holographic complexity. It is also possible to define the volume in the bulk as the maximal volume in the AdS which ends on the time slice at the AdS boundary, $V=V\left(\Sigma_{\max }\right)$ [24]. This would again leads to divergences, and so we will again need to regularize it. The latter can be achieved by subtracting the contribution of a background AdS $V(\gamma)$ AdS from the deformation of the AdS $V(\gamma)_{\text {AdS }}$, and defining

$\Delta \chi_{F}=\frac{V\left(\Sigma_{\max }\right)_{\mathrm{DAdS}}-V\left(\Sigma_{\max }\right)_{\mathrm{AdS}}}{8 \pi R G_{d+1}}$.

This quantity will be finite, and it will correspond to the fidelity susceptibility of a boundary field theory [25-27]. So, we shall call this quantity as the fidelity susceptibility even in the bulk. It has recently been proposed that the fidelity susceptibility has the same behavior as the thermodynamic volume in the extended phase space [28]. The cosmological constant is treated as the thermodynamics pressure in extended phase space, and it is possible to define a thermodynamic volume conjugate to this pressure [29-36]. In this paper, we will analyze the relation between the thermodynamic volume, fidelity susceptibility and holographic complexity for different black hole solutions. It may be noted that we will also use higher order corrections for the black hole solution previously studied, and hence more accurate results [28]. It will be observed that, for all these different black hole solutions, the thermodynamic volume has the same behavior as the fidelity susceptibility. Thus, the recently observed behavior for a specific black hole solution [28] seems to be a universal behavior of thermodynamic volume and fidelity susceptibility. Furthermore, it will be observed that the holographic complexity is different for all these cases. The information dual to a volume in the bulk is measured by the regularized fidelity susceptibility and the regularized holographic complexity [33,37-39], so this paper establishes a connection between the information dual to a volume and thermodynamics. We would like to point out that the original fidelity susceptibility and the original holographic complexity were divergences, and we have regularized them. Therefore, when we refer to the fidelity susceptibility and the holographic complexity, we are actually referring to these regularized fidelity susceptibility and regularized holographic complexity. It may also be noted that there are other proposals to calculate the holographic complexity of a system, and one of them relates the complexity to the action of the theory [40,41]. However, in this paper, we will define holographic complexity using the volume $V=V(\gamma)$ [23], and fidelity susceptibility using the volume $V=V\left(\Sigma_{\max }\right)$ [24].

\section{Schwarzschild-Anti-de Sitter black holes}

In this section, we consider black holes in AdS space as deformations that correspond to excited states on the CFT boundary. Then we calculate the temperature, entropy, and heat capacity. After that, we calculate $\Delta \mathcal{C}$ and $\Delta \chi_{F}$ by first calculating the volume of minimal surface of the AdS slice, and the maximal volume of that slice. It may be noted that in these calculations we use higher order corrections as compared to previous calculations done for similar black holes [28]. However, the real motivation to mention these improved calculations for Schwarzschild-Anti-de Sitter black holes is to review this case, and then use it for other black holes. We begin our study with a simple case where the geometry is a topological Schwarzschild-Anti-de Sitter (SAdS) black hole, whose metric is represented by the following line-element:

$\mathrm{d} s^{2}=-f(r) \mathrm{d} t^{2}+\frac{\mathrm{d} r^{2}}{f(r)}+r^{2} \mathrm{~d} \Omega^{2}$, 
where $\mathrm{d} \Omega^{2}=\mathrm{d} \theta^{2}+\frac{1}{k} \sin ^{2}(\sqrt{k} \theta) \mathrm{d} \phi^{2}$ with $k=\{-1,0,1\}$. Additionally, the metric function $f(r)$ is given by

$f(r)=1-\frac{2 M}{r}+\frac{r^{2}}{l^{2}}$.

By assuming that $r_{+}$is the black hole event horizon, i.e. $f\left(r_{+}\right)=0$, we can introduce a small parameter $\epsilon=\frac{M l^{2}}{r_{+}^{3}} \ll$

1 to rewrite the above function, yielding

$f=1+\frac{1}{l^{2}}\left(r^{2}-\frac{2 \epsilon r_{+}^{3}}{r}\right)$.

The mass and the volume of the sphere enclosed by the black hole are
$A=2(2 \pi) \int_{0}^{\theta_{0}} L(\theta) \mathrm{d} \theta$

Now, we will assume the following boundary conditions imposed on a minimal surface:

$r^{\prime}(0)=0, \quad r(0)=\rho$,

where $\rho$ is the turning point of the solution $r(\theta)$ and prime denotes differentiation with respect to $\theta$. The corresponding solution of the Euler-Lagrange equation for this Lagrangian density $L$ and the above boundary condition is given by

$r(\theta)=\rho-\frac{1}{2} \frac{\left(-\rho^{3}-\rho l^{2}+2 \epsilon r_{+}^{3}\right) \theta^{2}}{l^{2}}+\frac{1}{48} \frac{\left(18 \rho^{6}+28 \rho^{4} l^{2}-45 \epsilon r_{+}^{3} \rho^{3}+10 \rho^{2} l^{4}-29 \rho l^{2} \epsilon r_{+}^{3}+18 \epsilon^{2} r_{+}^{6}\right) \theta^{4}}{\rho l^{4}}$.

$M=\frac{r_{+}}{2}\left(1+\frac{r_{+}^{2}}{l^{2}}\right), \quad V=\frac{4}{3} \pi r_{+}^{3}$.

The temperature and specific heat of the black hole are, respectively,

$$
\begin{aligned}
T & =\frac{1}{4 \pi r_{+} l^{2}}\left(l^{2}+3 r_{+}^{2}\right), \\
c_{p} & =\left(\frac{\partial M}{\partial T}\right)_{p}=\frac{\frac{\partial M}{\partial r_{+}}}{\frac{\partial T}{\partial r_{+}}}=\frac{\frac{\partial}{\partial r_{+}}\left(\frac{r_{+}}{2}+\frac{r_{+}^{3}}{2 l^{2}}\right)}{\frac{\partial}{\partial r_{+}}\left(\frac{1}{4 \pi l^{2} r_{+}}\left(l^{2}+3 r_{+}^{2}\right)\right)} \\
& =2 \pi r_{+}^{2}\left(\frac{3 r_{+}^{2}+l^{2}}{3 r_{+}^{2}-l^{2}}\right) .
\end{aligned}
$$

Now, let us compute the area of the minimal surface $\gamma$, which is parametrized by $r=r(\theta)$ given by a time slice $t=0$ in the line-element,

$\left.\mathrm{d} s^{2}\right|_{t=0}=r^{2} \sin ^{2} \theta d \phi^{2}+\left(r^{2}+\frac{\left(\frac{\mathrm{d} r}{\mathrm{~d} \theta}\right)^{2}}{f(r)}\right) \mathrm{d} \theta^{2}$.

Hence, the minimal area can be written as follows:

Area $=\int_{0}^{2 \pi} \mathrm{d} \phi \int_{0}^{\theta_{0}} L(\theta) \mathrm{d} \theta$

where $L(\theta)=r \sin \theta \sqrt{r^{2}+\frac{\left(\frac{\mathrm{d} r}{\mathrm{~d}}\right)^{2}}{f(r)}}$ and $\theta_{0}$ is the upper boundary on the entangled domain. Therefore, the total area will be given by
Additionally, we obtain $L=-3\left(\rho r_{+}^{3} \theta^{3}\right) / l^{2}$ and hence, from (2.9), the total area can be expressed as

$A=-3 \pi \frac{\operatorname{csgn}(\rho) \rho r_{+}^{3} \theta_{0}^{4}}{l^{2}}$.

where

$\operatorname{csgn}(\rho)=\left\{\begin{array}{ll}1, & \rho>0 \\ -1, & \rho<0\end{array}\right.$.

Consequently, the regularized entanglement entropy (the difference of entanglement entropy between pure AdS and SAdS) with $\rho>0$ is

$\Delta S=-\frac{3}{16} \frac{\rho^{3} r_{+}^{3} \theta_{0}^{4}}{G l^{2}}$,

where $G$ is a gravitational constant.

In order to compute the holographic complexity, we need to evaluate the volume of the bulk enclosed by the same surface used in entanglement entropy. This volume is defined as follows:

$V=2 \pi \int_{0}^{\theta_{0}} \mathrm{~d} \theta \int_{r_{+}}^{r(\theta)} \frac{r^{2} \mathrm{~d} r}{\sqrt{f(r)}}$.

In order to solve the integral in the above expression, we can expand the integrand in Taylor series of the parameter $\epsilon$ as

$$
\frac{r^{2}}{\sqrt{f(r)}}=\frac{r^{2}}{\sqrt{\frac{l^{2}+r^{2}}{l^{2}}}}+\frac{r r_{+}^{3}}{\sqrt{\frac{l^{2}+r^{2}}{l^{2}}}\left(l^{2}+r^{2}\right)} \epsilon+\mathcal{O}\left(\epsilon^{2}\right) .
$$


Then, by leaving only linear terms in $\epsilon$ and expanding in $\theta$ up to second order we find that the volume is

$\Delta V=\frac{1}{2}\left(-\frac{r_{+}^{3} l}{\sqrt{l^{2}+\rho^{2}}}+\frac{r_{+}^{3} l}{\sqrt{l^{2}+r_{+}^{2}}}\right) \theta_{0}^{2}$.

Now, using the above volume, we can finally compute the holographic complexity, which is

$$
\Delta \mathcal{C}=\frac{\Delta V}{8 \pi l}=\frac{1}{16 \pi l}\left(-\frac{r_{+}^{3} l}{\sqrt{l^{2}+\rho^{2}}}+\frac{r_{+}^{3} l}{\sqrt{l^{2}+r_{+}^{2}}}\right) \theta_{0}^{2},
$$

where $\Delta V=V_{S A d S}-V_{\text {AdS }}$. Now as we are going to analyze the $P-V$ relation for this system, we define this regularized volume as volume associated with holographic complexity $\Delta V=V_{c}$, and define the complexity pressure $P_{c}$ as the thermodynamic pressure associated with $V_{c}$, as $P=-\frac{\partial M}{\partial V_{c}}$, yielding

$$
\begin{aligned}
P_{c} & =-\frac{\frac{\partial M}{\partial r_{+}}}{\frac{\partial V_{c}}{\partial r_{+}}} \\
& =-\frac{\left(1+3 r_{+}^{2}\right) \sqrt{1+\rho^{2}}\left(1+r_{+}^{2}\right)^{3 / 2}}{r_{+}^{2}\left(-3 \sqrt{1+r_{+}^{2}} r_{+}^{2}-3 \sqrt{1+r_{+}^{2}}+2 r_{+}^{2} \sqrt{1+\rho^{2}}+3 \sqrt{1+\rho^{2}}\right) \theta_{0}^{2}},
\end{aligned}
$$

where the mass of black hole is given in (2.4). Moreover, from (2.4), we can express the event horizon of the black hole in terms of the temperature, obtaining

$r_{+}=\frac{2}{3} \pi T \pm \frac{1}{3} \sqrt{4 \pi^{2} T^{2}-3}$.

It is important to remark that we will choose the root with the minus sign since only this root has a physical meaning for the case where $k>1$. Now, by using the above expression, we can rewrite the volume and the complexity pressure given by (2.18) and (2.19) in terms of the temperature. Note that the explicit expressions are found in Appendix A. Since both functions $P, V$ are functions of the temperature $T$ and the parameter $\theta_{0}$, it is illustrative to plot these functions as contour plots. These figures are displayed in Figs. 1, 2. In each graph we fixed the temperature $T$ and consequently each function $P, V$ are defined as a single variable function of $\theta_{0}$. Since $V$ and $P$ depend on $\theta_{0}$, it is also possible to rewrite $P=P(T, V)$ as an equation of state if we eliminate $\theta_{0}$ among these two expressions for $P$ and $V$. and rewrite the complexity volume and pressure in terms of the temperature. Another volume dual to the thermodynamical volume in the bulk could be the maximum volume which is proportional to

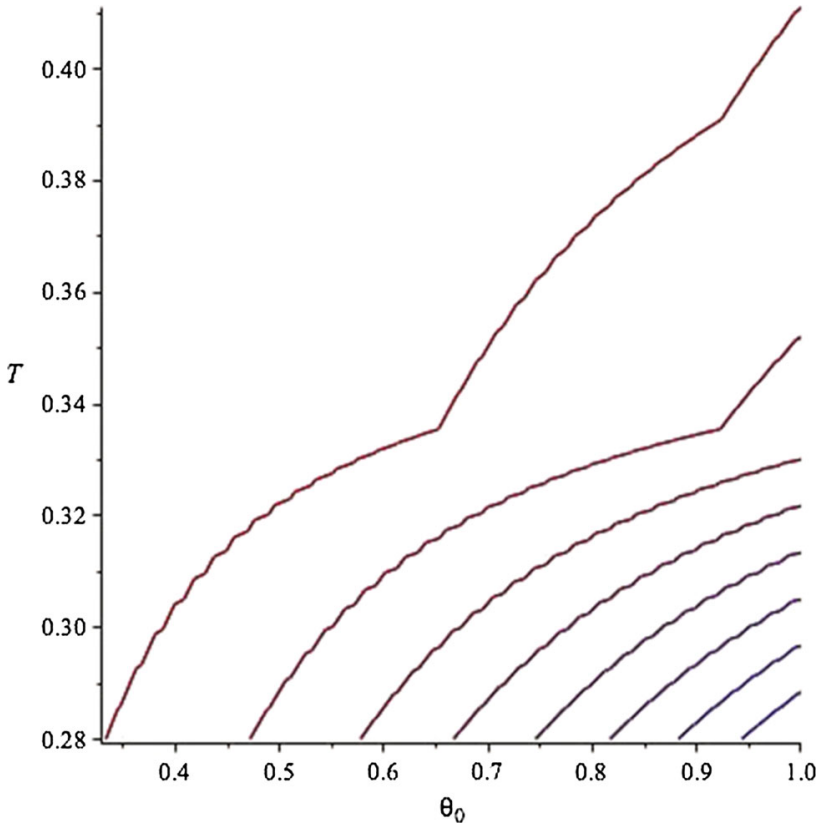

Fig. 1 Figures show contour plots of $T=$ constant in $V$ as functions of $\theta_{0}$. Different lines in each graph show a specific temperature

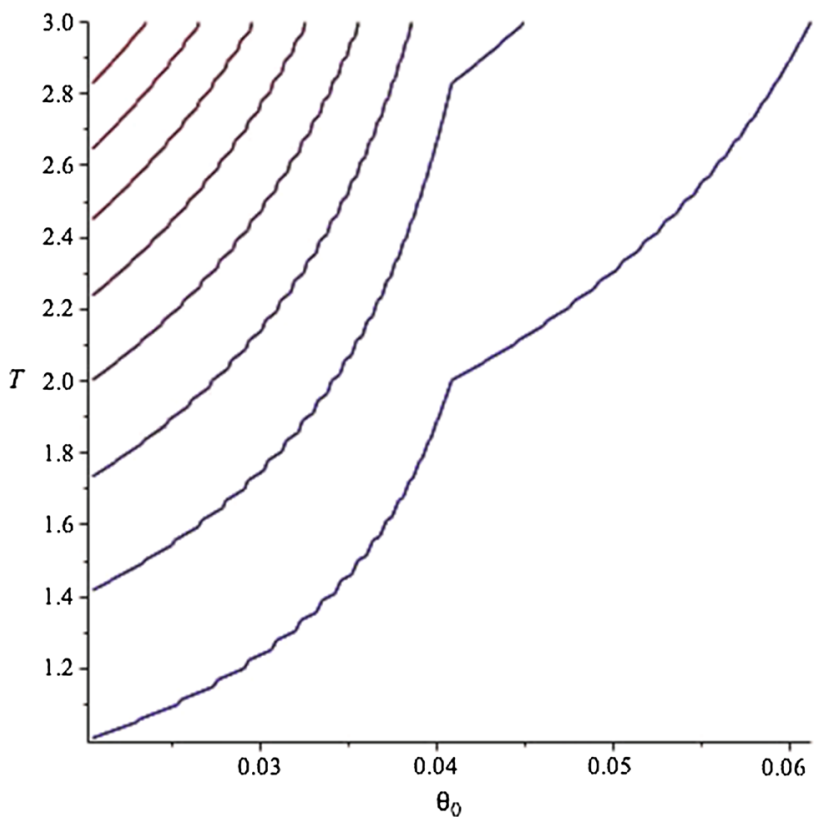

Fig. 2 Figures show contour plots of $T=$ constant in $P$ as functions of $\theta_{0}$. Different lines in each graph show a specific temperature

the fidelity susceptibility in the dual CFT part. The latter is defined as follows:

$\Delta \chi_{F}=\frac{V_{\max }}{8 \pi l G}$.

where $l$ is the AdS radius and $G$ the Newtonian constant. To evaluate $V_{\max }$, we should subtract the pure AdS background 
portion. We can expand in series the metric function because $\epsilon=0$ corresponds to the AdS background.

$$
\frac{1}{\sqrt{f}}=\frac{1}{\sqrt{f_{0}+\epsilon \delta f}} \simeq \frac{1}{\sqrt{f_{0}}}\left(1-\frac{\epsilon \delta f}{2 f_{0}}\right) .
$$

After a simple algebraic manipulation we find

$$
\begin{aligned}
V_{\mathrm{Fid}}= & V_{\mathrm{AdS}}+\epsilon \Delta V_{\mathrm{Fid}} \\
= & \int_{r_{+}}^{r_{\infty}} \frac{r d r}{\sqrt{f_{0}}}\left(1-\frac{\epsilon \delta f}{2 f_{0}}\right) \simeq \infty \\
& -\frac{\epsilon}{2} \int_{r_{+}}^{r_{\infty}} \frac{r \delta f}{f_{0}}{ }^{3 / 2} \mathrm{~d} r .
\end{aligned}
$$

Using the fact that $f_{0}=1+\frac{r^{2}}{l^{2}}$ (the metric of pure AdS) and $\delta f=-\frac{2 r_{+}^{3}}{r}$, the corresponding volume is

$$
\begin{aligned}
\Delta V_{\mathrm{Fid}}= & -\frac{r_{+}^{3}}{8\left(1+2 r_{+}^{2}+r_{+}^{4}\right)} \\
& {\left[10 r_{+}+6 \arctan \left(r_{+}\right)+6 \arctan \left(r_{+}\right) r_{+}^{4}\right.} \\
& \left.+6 r_{+}^{3}+12 \arctan \left(r_{+}\right) r_{+}^{2}-3 \pi-6 \pi r_{+}^{2}-3 \pi r_{+}^{4}\right],
\end{aligned}
$$

and the pressure reads

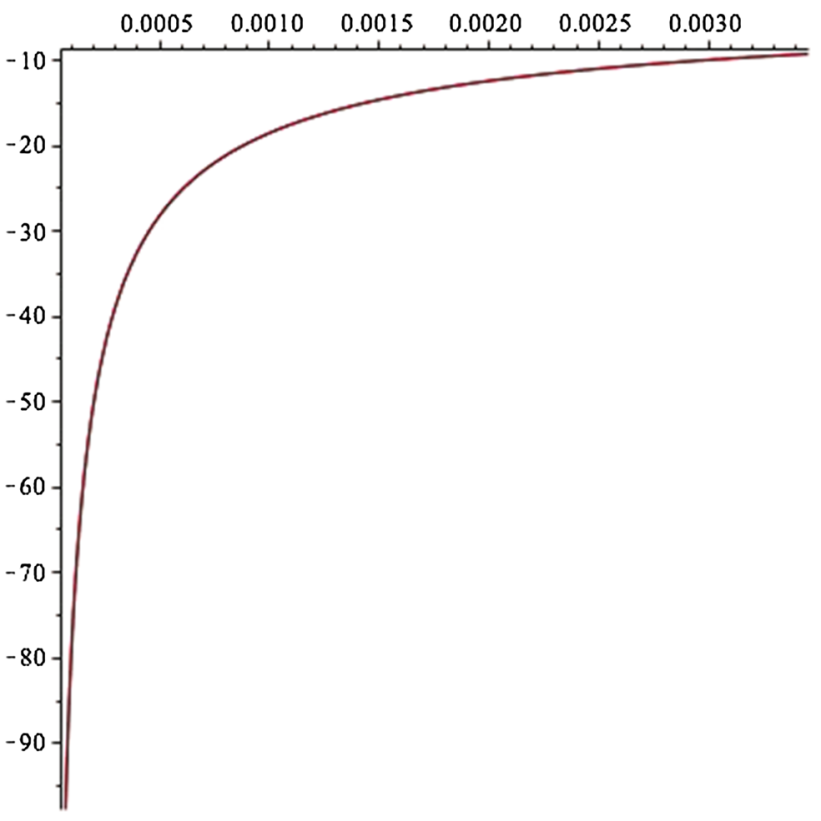

Fig. 3 This is a parametric plot for fidelity pressure and volume. This graph shows a fidelity-based equation of state as an attempt to find holographic version of thermodynamics

process. In the next section, we shall obtain the same calculation for a different deformation of AdS, with $U(1)$ charged black holes.

$$
P_{\mathrm{Fid}}=-\frac{\partial M}{\partial V_{\mathrm{Fid}}}=-\frac{\frac{\partial M}{\partial r_{+}}}{\frac{\partial V_{\mathrm{Fid}}}{\partial r_{+}}}=-\frac{\frac{4}{3}\left(\frac{1}{3}+r_{+}^{2}\right)\left(1+r_{+}^{2}\right)^{3}}{r_{+}^{2}\left(-2\left(1+r_{+}^{2}\right)^{3} \arctan \left(r_{+}\right)+\pi r_{+}^{6}-\frac{16}{3} r_{+}^{3}-2 r_{+}^{5}+3 \pi r_{+}^{2}+3 \pi r_{+}^{4}+\pi-\frac{46}{9} r_{+}\right)} .
$$

Note that $r_{+}$is given by Eq. (2.20). The parametric plot of $p=p(V, T)$ is depicted in Fig. 3. Here the horizontal line corresponds to $V$ and the vertical line to $p$.

Thus, we have calculated the holographic complexity and fidelity susceptibility from the associated minimal surface, and maximal volumes, respectively, and obtained a thermodynamic equation of states for SAdS black holes. We were also able to obtain the $P-V$ graphs for these quantities, which are displayed in Figs. 1, 2, 3, 4, 5, 6. It was observed that the $P-V$ relation for the fidelity susceptibility could be related to the $P-V$ relation for the thermodynamic volume and pressure in extended phase space, where the cosmological constant of the AdS space is viewed as a thermodynamic pressure $[29,32]$. It may be noted that the dual theory to Schwarzschild-AdS has been studied [42]. It would be possible to study the fidelity susceptibility of such a dual theory, and thus analyze the behavior of fidelity susceptibility. As this is the behavior obtained from a well defined field theory, we expect it to be unitary. Now as the black hole thermodynamics in extended phase space shows the same behavior, we expect the black hole thermodynamics to also be a unitary

\section{Reissner-Nordström-Anti-de Sitter black holes (RNSAdS)}

The aim of this section is to extend the previous section to a charged AdS black hole background. We will obtain fidelity susceptibility and holographic complexity and holographic equations of state.

The metric for RNSAdS is given by Eq. (2.1) with the function $f$ being

$f=1+\frac{r^{2}}{l^{2}}-\frac{\epsilon r_{+}^{3}}{r l^{2}}+\frac{\delta r_{+}^{4}}{r^{2} l^{2}}$,

where $\epsilon$ and $\delta$ are defined as

$$
\frac{2 M l^{2}}{r_{+}^{3}}=\epsilon, \quad \frac{Q^{2} l^{2}}{r_{+}^{4}}=\delta .
$$

Here, we also have $|Q|<l / 6$ and correspondingly $\delta<$ $l^{2} /\left(6 r_{+}^{2}\right)$. Let us start to compute extremal surfaces using the area functional. The area functional for a specific entangled 


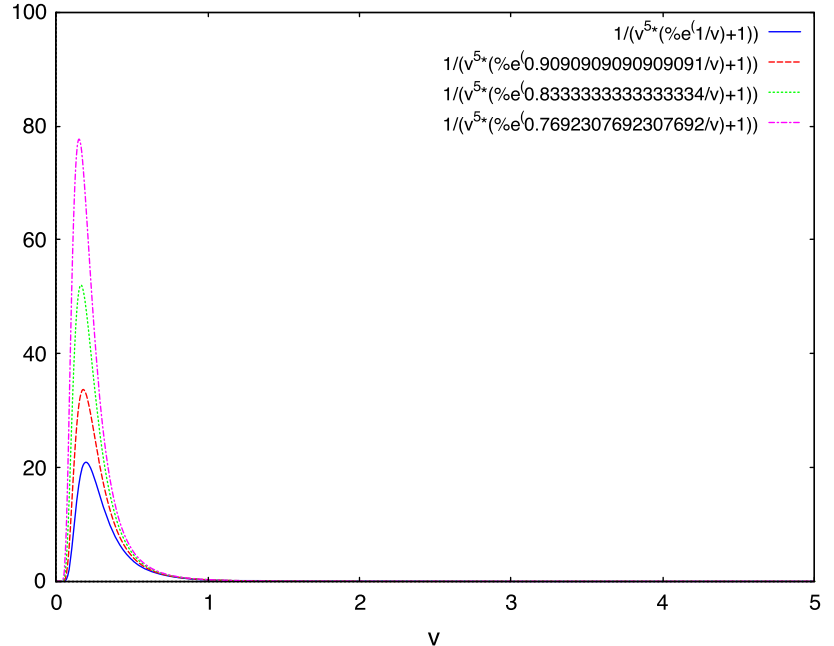

Fig. $4 P-V$ diagram for the thermodynamic volume given by $V=$ $\frac{4}{3} \pi r_{+}^{3}$ and pressure $P=\frac{3}{8 \pi l^{2}}$ for SAdS black holes. Here from bottom to top $T=\frac{1}{2} \frac{\sqrt{3}}{\pi}$ (blue), $T=\frac{1}{2} \frac{\sqrt{4}}{\pi}$ (red), $T=\frac{1}{2} \frac{\sqrt{5}}{\pi}$ (green), $T=\frac{1}{2} \frac{\sqrt{6}}{\pi}$ (pink)

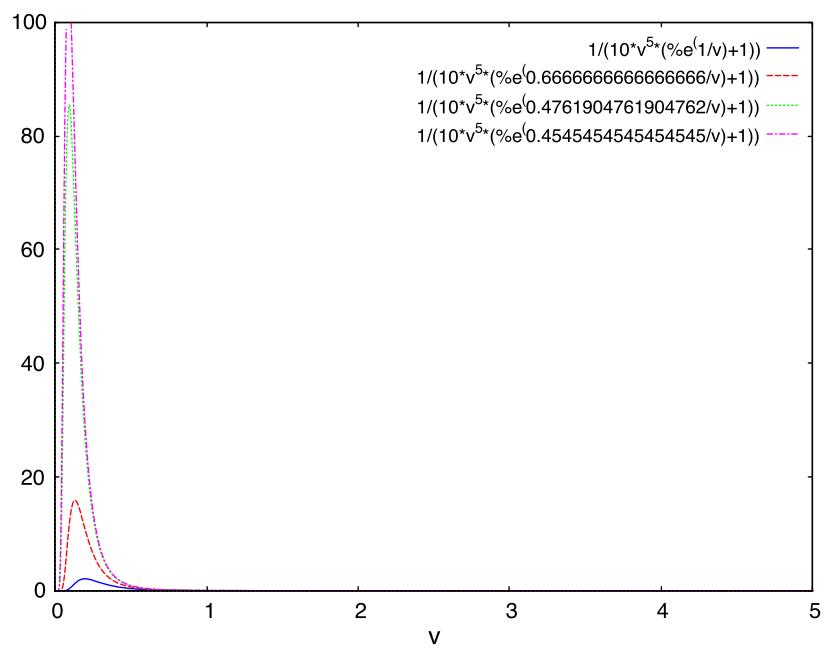

Fig. $5 P-V$ diagram of fidelity versus pressure. We address various temperatures of SAdS black holes. We indicate that fidelity does indeed represent thermodynamic volume. Here from bottom to top $T=\frac{1}{2} \frac{\sqrt{3}}{\pi}$ (blue), $T=\frac{1}{2} \frac{\sqrt{4}}{\pi}$ (red), $T=\frac{1}{2} \frac{\sqrt{5}}{\pi}$ (green), $T=\frac{1}{2} \frac{\sqrt{6}}{\pi}$ (pink)

region of the boundary in RNSAdS is then given by Eq. (2.8) where $L(\theta)$ now is

$L(\theta)=r \sin \theta \sqrt{r^{2}+\frac{\left(\frac{d r}{d \theta}\right)^{2}}{1+\frac{r^{2}}{l^{2}}-\frac{\epsilon r_{+}^{3}}{r l^{2}}+\frac{\delta r_{+}^{4}}{r^{2} l^{2}}}}$.

The Euler-Lagrange equation corresponding to the above $L(\theta)$ is written in the appendix (see B.1). We can solve this equation by expanding in series of $\theta$. Doing this, up to sixth order in $\theta$ we find (we set here the AdS radius $l=1$ )

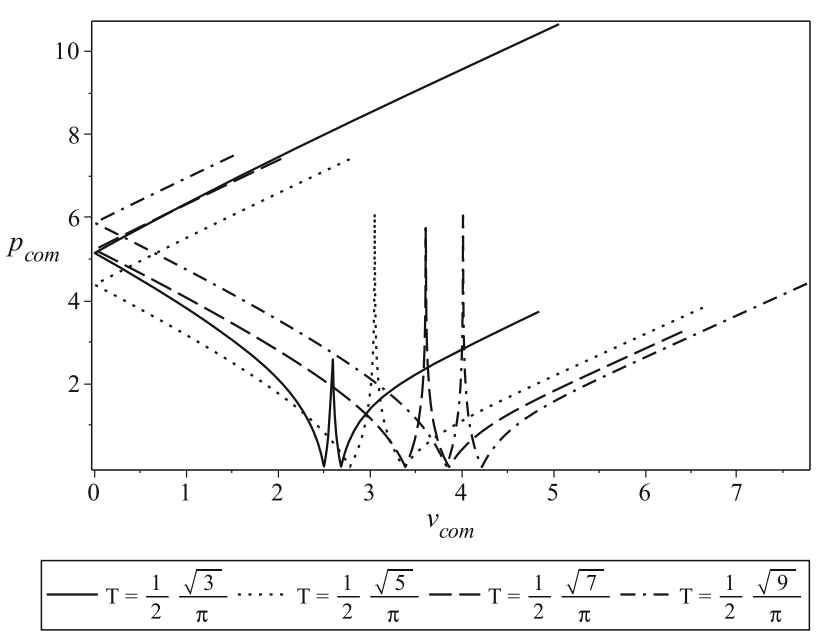

Fig. 6 A $P-V$ diagram between holographic complexity and pressure, showing a totally different behavior than the thermodynamic $P-V$ diagram

$$
\begin{aligned}
r(\theta)= & \rho+\frac{1}{2} \frac{\left(-\rho \epsilon r_{+}^{3}+\delta r_{+}^{4}+\rho^{4}+\rho^{2}\right) \theta^{2}}{\rho} \\
& +\frac{1}{96 \rho^{2}}\left[\left(9 \rho \epsilon^{2} r_{+}^{6}-9 \epsilon h^{7} \delta-45 \rho^{4} \epsilon r_{+}^{3}-29 \rho^{2} \epsilon r_{+}^{3}\right.\right. \\
& \left.\left.+36 \rho^{3} \delta r_{+}^{4}+20 \rho \delta r_{+}^{4}+36 \rho^{7}+56 \rho^{5}+20 \rho^{3}\right) \theta^{4}\right] \\
& +\mathcal{O}\left(\theta^{6}\right) .
\end{aligned}
$$

Now, by expanding the above expression in $\theta$ up to sixth order, and then again expanding it in $\delta$ up to second order, we find that the finite part of the entanglement entropy. This part is the difference between the pure background and the AdS deformation of the metric. Doing that, we find

$$
\begin{aligned}
\Delta S= & -\frac{1440 \rho}{4 G} \times\left[\theta _ { 0 } ^ { 2 } \left(675 \theta_{0}^{4} r_{+}^{3} \epsilon \rho^{4}+540 \theta_{0}^{2} r_{+}^{3} \epsilon \rho^{2}\right.\right. \\
& +375 \theta_{0}^{4} r_{+}^{7} \epsilon \delta+495 \theta_{0}^{4} r_{+}^{3} \epsilon \rho^{2}-450 \theta_{0}^{4} \rho^{7} \\
& -272 \theta_{0}^{4} \rho^{3}-720 \theta_{0}^{4} \rho^{5}-540 \rho^{5} \theta_{0}^{2} \\
& -480 \rho^{3} \theta_{0}^{2}-420 \theta_{0}^{4} \rho \delta r_{+}^{4}-600 \theta_{0}^{4} \rho^{3} \delta r_{+}^{4} \\
& \left.\left.-720 \rho^{3}-540 \rho \theta_{0}^{2} \delta r_{+}^{4}\right)\right]^{-1}
\end{aligned}
$$

The complete expressions for $L(\theta)$ and the integral related with the area are displayed in Appendix B.1 (see Eqs. (B.5) and (B.6)).

Let us now compute the holographic complexity and fidelity susceptibility dual volumes for a RN black hole. These quantities can be written as follows:

$$
\begin{aligned}
V_{c} & =2 \pi \int_{0}^{\theta_{0}} \sin \theta \mathrm{d} \theta \int_{r_{+}}^{r(\theta)} \frac{r^{2} \mathrm{~d} r}{\sqrt{f}}, \\
V_{\text {Fid }} & =2 \pi \int_{0}^{2 \pi} \sin \theta \mathrm{d} \theta \int_{r_{+}}^{r_{\infty}} \frac{r^{2} \mathrm{~d} r}{\sqrt{f}} .
\end{aligned}
$$


Now, by expanding the integrand $r^{2} / \sqrt{f}$ in Taylor series up to linear terms in $\epsilon$ and $\theta$, we obtain

$$
\begin{aligned}
& \frac{r^{2}}{\sqrt{1+\frac{r^{2}}{l^{2}}-\frac{\epsilon r_{+}^{3}}{r l^{2}}+\frac{\delta r_{+}^{4}}{r^{2} l^{2}}}} \\
& =-\frac{1}{4} \frac{l r_{+}^{3}\left(-2 r^{2} \epsilon l^{2}-2 r^{4} \epsilon+2 r+\delta r l^{2}+2 r_{+} \delta r^{3}+3 r_{+}^{4} \delta \epsilon\right)}{r\left(l^{2}+r^{2}\right)^{5 / 2}} .
\end{aligned}
$$

Hence, the volume corresponding to the holographic complexity of the RN black hole becomes $(l=1)$

$$
\begin{aligned}
V_{c}= & -\int_{0}^{\theta_{0}} \sin \theta \mathrm{d} \theta\left(\frac{1}{2} \frac{r_{+}^{3} \epsilon r^{2}}{\left(1+r^{2}\right)^{3 / 2}}\right. \\
& +\frac{1}{2} \frac{r_{+}^{3} \epsilon}{\left(1+r^{2}\right)^{3 / 2}}+\frac{1}{2} \frac{\delta r_{+}^{4} r}{\sqrt{1+r^{2}}}+\frac{1}{4} \frac{\epsilon r_{+}^{7} \delta}{\left(1+r^{2}\right)^{3 / 2}} \\
& \left.+\frac{3}{4} \frac{\epsilon r_{+}^{7} \delta}{\sqrt{1+r^{2}}}-\frac{3}{4} \epsilon r_{+}^{7} \delta \ln \left[\frac{2+2 \sqrt{1+r^{2}}}{r}\right]\right)\left.\right|_{r_{+}} ^{r(\theta)} .
\end{aligned}
$$

After computing this integral and expanding up to sixth order in $\theta$, we find Eq. (B.7). After some mathematical steps (explained in Appendix B.1), by taking an asymptotic expansion in $\rho$ we find the following compacted expression:

$$
\begin{aligned}
V_{c}= & -\frac{1}{48} \frac{\left(-6 \theta_{0}^{2} \epsilon b r_{+}^{2}-6 \theta_{0}^{2} \epsilon b\right) \theta_{0}^{2} r_{+}^{3} \pi \rho}{b^{3}} \\
& -\frac{1}{48 b^{3}} \times\left[-3 \theta_{0}^{2} r_{+}^{6} \delta b \epsilon \ln \left(\frac{1+b}{r_{+}}\right)\right. \\
& +2 \theta_{0}^{2} r_{+}^{4} \delta+4 \theta_{0}^{2} r_{+}^{3} \delta b-48 r_{+}^{4} \delta \epsilon-24 r_{+}^{4} \delta \\
& +2 \theta_{0}^{2} \epsilon r_{+}^{2}+4 \theta_{0}^{2} r_{+} \delta b \\
& +24 r_{+} \delta b-3 \theta_{0}^{2} r_{+}^{4} \delta b \epsilon \ln \left(\frac{1+b}{r_{+}}\right) \\
& +2 \theta_{0}^{2} r_{+}^{2} \delta+4 \theta_{0}^{2} r_{+}^{4} \delta \epsilon \\
& -24 \epsilon+2 \theta_{0}^{2} \epsilon-24 \epsilon r_{+}^{2}+24 r_{+}^{3} \delta b-36 r_{+}^{6} \delta \epsilon \\
& +36 r_{+}^{4} \delta \epsilon b \ln \left(\frac{1+b}{r_{+}}\right)-24 r_{+}^{2} \delta \\
& \left.+36 r_{+}^{6} \delta \epsilon b \ln \left(\frac{1+b}{r_{+}}\right)+3 \theta_{0}^{2} r_{+}^{6} \delta \epsilon\right] \theta_{0}^{2} r_{+}^{3} \pi
\end{aligned}
$$

where $a=\sqrt{1+\rho^{2}}$ and $b=\sqrt{1+r_{+}^{2}}$.
The mass, temperature and complexity pressure of the RN black hole are defined as follows:

$$
\begin{aligned}
& M=\frac{r_{+}}{2}\left(1+\frac{r_{+}^{2}}{l^{2}}+\frac{Q^{2}}{r_{+}^{2}}\right), \\
& T=\frac{1}{4 \pi}\left(\frac{3 r_{+}^{4}+r_{+}^{2}-Q^{2}}{r_{+}^{3}}\right), \\
& P=-\frac{\partial M}{\partial V_{\mathrm{Fid}}}=-\frac{\frac{\partial M}{\partial r_{+}}}{\frac{\partial V_{\mathrm{Fid}}}{\partial r_{+}}} .
\end{aligned}
$$

The explicit expression for the complexity pressure is very long for the spacetime studied. The complete and expanded expressions are displayed in Appendix B.1. Now, we need to express the complexity pressure and volume in terms of the temperature. In order to do that, we need to solve (3.12) for $r_{+}$. Thus, we need to solve the following equation:

$r_{+}^{4}-\frac{4 \pi T}{3} r_{+}^{3}+\frac{r_{+}^{2}}{3}-\frac{Q^{2}}{3}=0$.

The roots of this equation are given by

$$
\begin{aligned}
& x_{1,2}=-\frac{\tilde{b}}{4 \tilde{a}}-S \pm \frac{1}{2} \sqrt{-4 S^{2}-2 p+\frac{q}{S}} \\
& x_{3,4}=-\frac{\tilde{b}}{4 \tilde{a}}+S \pm \frac{1}{2} \sqrt{-4 S^{2}-2 p+\frac{q}{S}}
\end{aligned}
$$

where $p, q$ and $S$ are defined by

$$
\begin{aligned}
p & =\frac{8 \tilde{a} \tilde{c}-3 \tilde{b}^{2}}{8 \tilde{a}^{2}}, \quad q=\frac{\tilde{b}^{3}-4 \tilde{a} \tilde{b}+8 \tilde{a}^{2} \tilde{d}}{8 \tilde{a}^{3}} \\
S & =\frac{1}{6} \sqrt{-6+3 G+3 \frac{\Delta_{0}}{G}}, \quad G=\sqrt[3]{\frac{1}{2} \Delta_{1}+\frac{1}{2} \sqrt{\Delta_{1}^{2}-4 \Delta_{0}^{3}}} \\
\Delta_{0} & =\tilde{c}^{2}-3 \tilde{b} \tilde{d}+12 \tilde{a} \tilde{e}, \\
\Delta_{1} & =2 \tilde{c}^{3}-9 \tilde{b} \tilde{c} \tilde{d}+27 \tilde{b}^{2} \tilde{e}+27 \tilde{a} \tilde{d}^{2}-72 \tilde{a} \tilde{c} \tilde{e}
\end{aligned}
$$

and $\Delta$, determined by

$\Delta_{1}^{2}-4 \Delta_{0}^{3}=-27 \Delta$

is a determinant of the fourth order polynomial. If $\Delta>0$, then all four roots of the equation are either real or complex. From (3.14), we have $\tilde{a}=1, \tilde{b}=-(4 \pi T) / 3, \tilde{c}=1 / 3$, $\tilde{d}=0$ and $\tilde{e}=-Q^{2} / 3$. Therefore, for our case the roots are given by 


$$
\begin{aligned}
r_{+}^{1,2}= & \frac{1}{3} \pi T-\frac{1}{6} \sqrt{-6+3 \sqrt[3]{k}+3 \frac{\frac{1}{9}-4 Q^{2}}{\sqrt[3]{k}}} \\
& \pm \frac{1}{6} \sqrt{-3 \sqrt[3]{k}-3 \frac{\frac{1}{9}-4 Q^{2}}{\sqrt[3]{k}}+12 \pi^{2} T^{2}+\frac{54\left(-\frac{8}{27} \pi^{3} T^{3}+\frac{2}{3} \pi T\right)}{\sqrt{-6+3 \sqrt[3]{k}+3 \frac{1 / 9-4 Q^{2}}{\sqrt[3]{k}}}}}, \\
r_{+}^{3,4}= & \frac{1}{3} \pi T-\frac{1}{6} \sqrt{-6+3 \sqrt[3]{k}+3 \frac{\frac{1}{9}-4 Q^{2}}{\sqrt[3]{k}}} \\
& \pm \frac{1}{6} \sqrt{-3 \sqrt[3]{k}-3 \frac{\frac{1}{9}-4 Q^{2}}{\sqrt[3]{k}}+12 \pi^{2} T^{2}+\frac{54\left(-\frac{8}{27} \pi^{3} T^{3}+\frac{2}{3} \pi T\right)}{\sqrt{-6+3 \sqrt[3]{k}+3 \frac{1 / 9-4 Q^{2}}{\sqrt[3]{k}}}}},
\end{aligned}
$$

where

$$
\begin{aligned}
k= & \frac{1}{27}-8 \pi^{2} T^{2} Q^{2}+4 Q^{2} \\
& +\frac{1}{2} \sqrt{\left(\frac{2}{27}-16 \pi^{2} T^{2} Q^{2}+8 Q^{2}\right)^{2}-4\left(\frac{1}{9}-4 Q^{2}\right)^{3}} .
\end{aligned}
$$

Since $k$ must be real, the inequality

$$
\left(\frac{2}{27}-16 \pi^{2} T^{2} Q^{2}+8 Q^{2}\right)^{2}-4\left(\frac{1}{9}-4 Q^{2}\right)^{3} \geq 0
$$

must hold. Equivalently, the above inequality can be expressed as

$$
T \leq \frac{1}{4 Q \pi} \sqrt{-\left(2\left(\frac{1}{9}-4 Q^{2}\right)^{3 / 2}-\frac{2}{27}-8 Q^{2}\right)} .
$$

Now, by taking series in $Q$ up to order six in the above equation, we find

$$
T \leq \frac{1}{2} \frac{\sqrt{3}}{\pi}-\frac{3}{4} \frac{\sqrt{3} Q^{2}}{\pi}-\frac{81}{16} \frac{\sqrt{3} Q^{4}}{\pi}+\mathcal{O}\left(Q^{6}\right),
$$

or

$T \leq 0.27567-0.41350 Q^{2}-2.7912 Q^{4}+\mathcal{O}\left(Q^{6}\right)$.

From this expression (see Fig. 7), we note that the temperature will be maximum when $Q=0$. Let us now find the horizon of the RNSAdS black hole. To do that, we need to rewrite (3.1) using (3.2), which gives us

$f(r)=1+\frac{r^{2}}{l^{2}}-\frac{2 M}{r}+\frac{Q^{2}}{r^{2}}$.
Now, the horizon condition $f(r)=0$ can be written as

$\xi^{4}+\xi^{2}-\frac{2 M}{l} \xi+\left(\frac{Q}{l}\right)^{2}=0$,

where $\xi=r / l$. Here our aim is to find the largest root of the above equation which will correspond to the outer horizon of the RN charged black hole. The largest root is given by

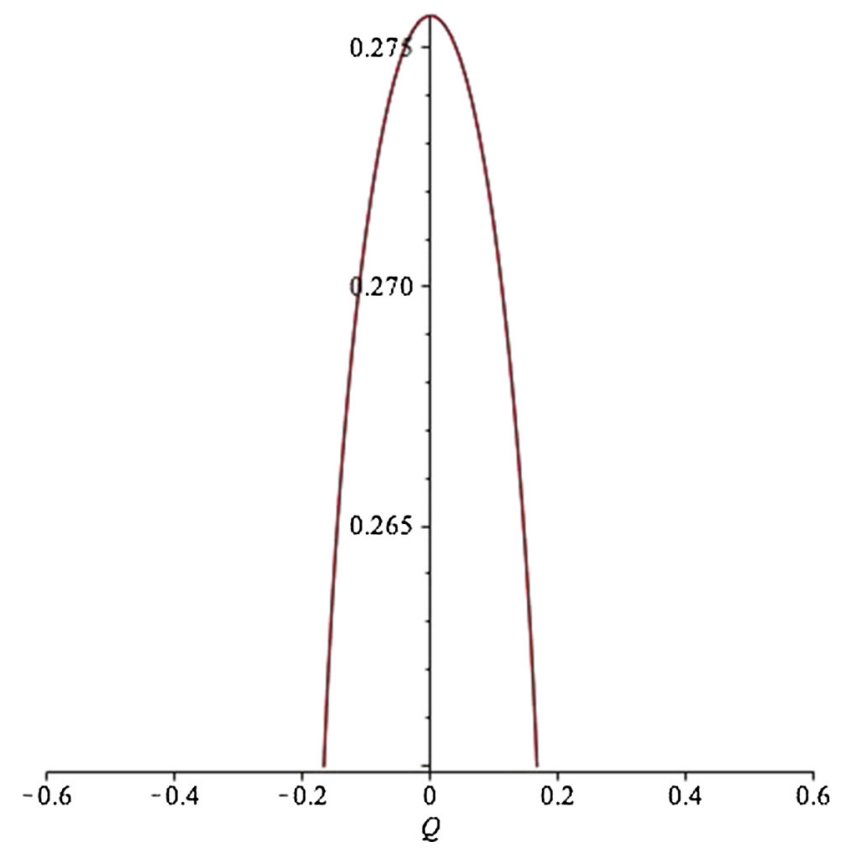

Fig. $7 T$ as a function of $Q$. This graph is confined with the condition $|Q|<\frac{l}{6}$ 


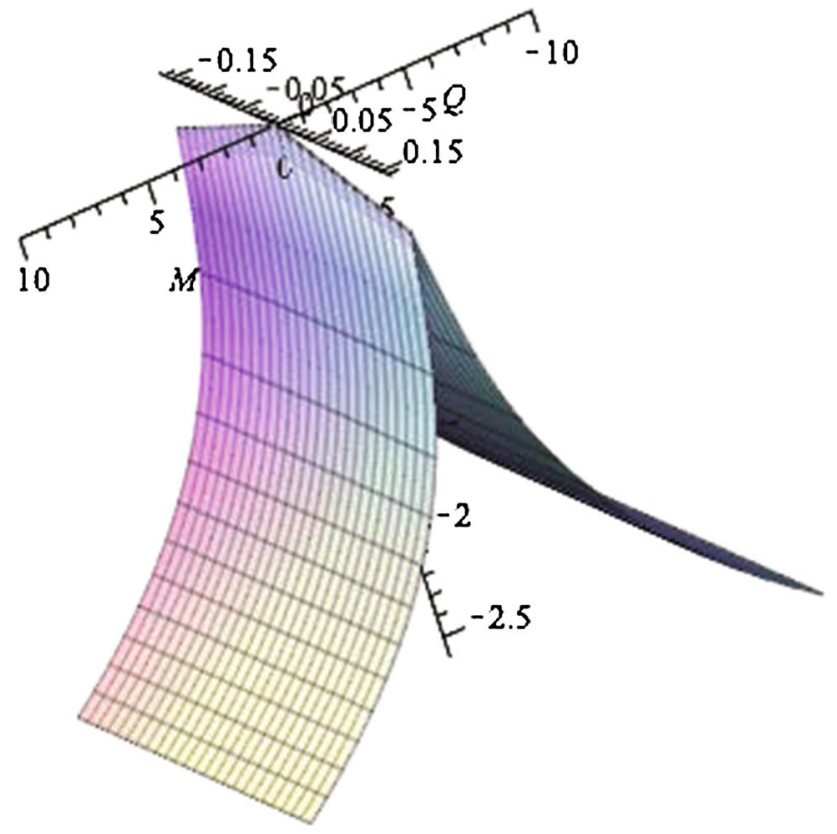

Fig. 8 Location of the roots $\xi_{1}$ for the RN black hole as a function of $Q, M$

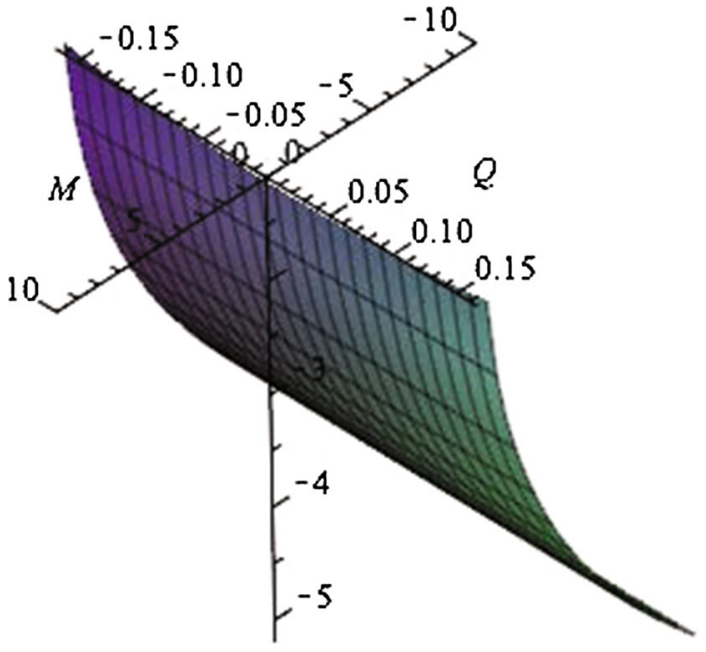

Fig. 9 Location of the roots $\xi_{2}$ for the RN black hole as a function of $Q, M$

$$
\begin{aligned}
\xi_{3}= & \frac{1}{6}\left(-6+3 k+3 \frac{1+12 Q^{2}}{k}\right)^{1 / 2} \\
& +\frac{1}{6} \sqrt{-12-3 m-\frac{3\left(1+12 Q^{2}\right)}{m}-\frac{108 M}{\sqrt{-6+3 m+3 \frac{1+12 Q^{2}}{m}}}},
\end{aligned}
$$

where $m=\left(1+54 M^{2}+6\right.$

$\left.\sqrt{3 M^{2}+81 M^{4}-Q^{2}-12 Q^{4}-48 Q^{6}}\right)^{1 / 3}$. This root can be seen in Figs. 8, 9 and 10.

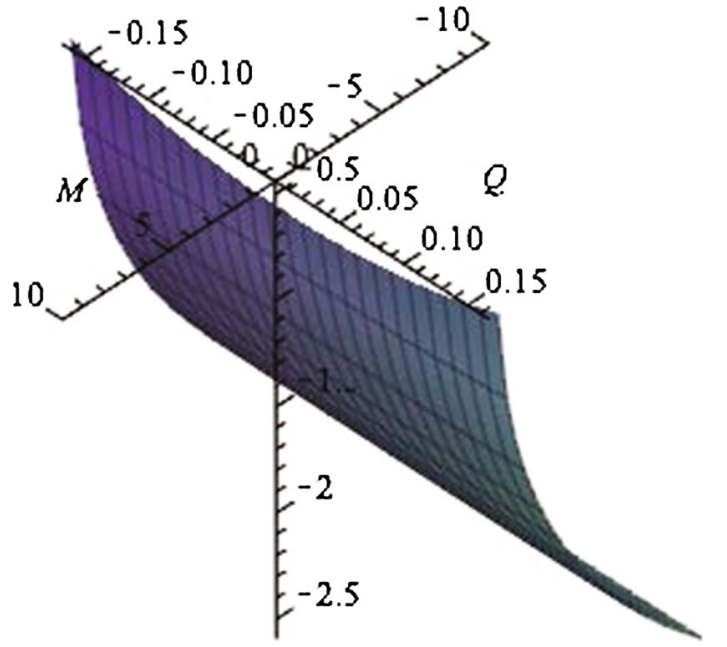

Fig. 10 Location of the roots $\xi_{3}$ for the RN black hole as functions of $Q, M$. We show that $\xi_{3}$ is the largest root

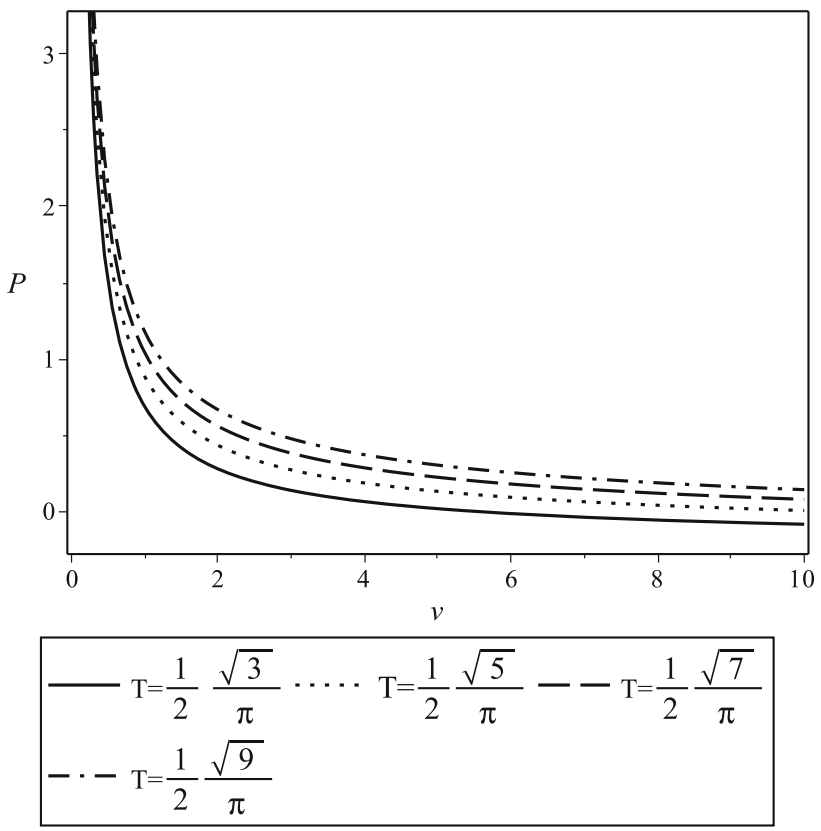

Fig. $11 P-V$ diagram for the thermodynamic volume $V=\frac{4}{3} \pi r_{+}^{3}$ and pressure $P=\frac{3}{8 \pi l^{2}}$ for RNSAdS black holes

From Figs. 11 and 12, we again observe that he $P-V$ behavior of fidelity susceptibility is similar to the $P-V$ relation of thermodynamic volume and pressure. However, in Fig. 13, we observe that the $P-V$ relation obtained from holographic complexity is very different. The dual to a ReissnerNordström-AdS black hole has been studied [44,45], and it is possible to obtain the fidelity susceptibility of this dual theory. This should correspond to the fidelity susceptibility calculated from the bulk. Now as the behavior of fidelity susceptibility from the dual theory cannot break unitarity, the behavior of fidelity susceptibility in the bulk cannot also 


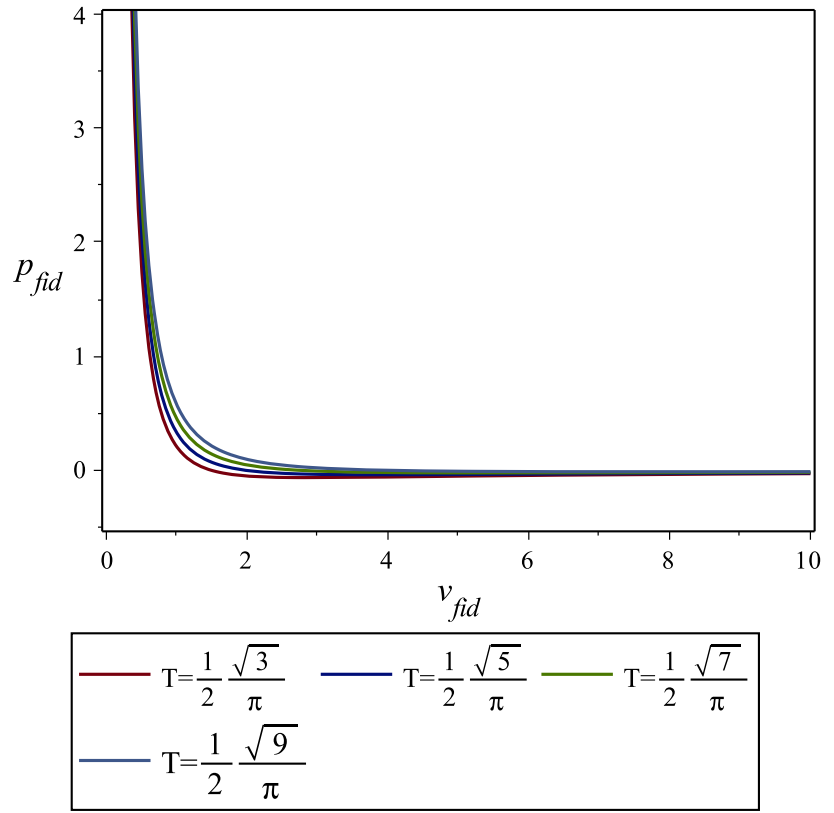

Fig. $12 P-V$ diagrams for fidelity versus pressure. We address various temperatures of RNSAdS black holes. We indicate that fidelity does indeed represent thermodynamic volume

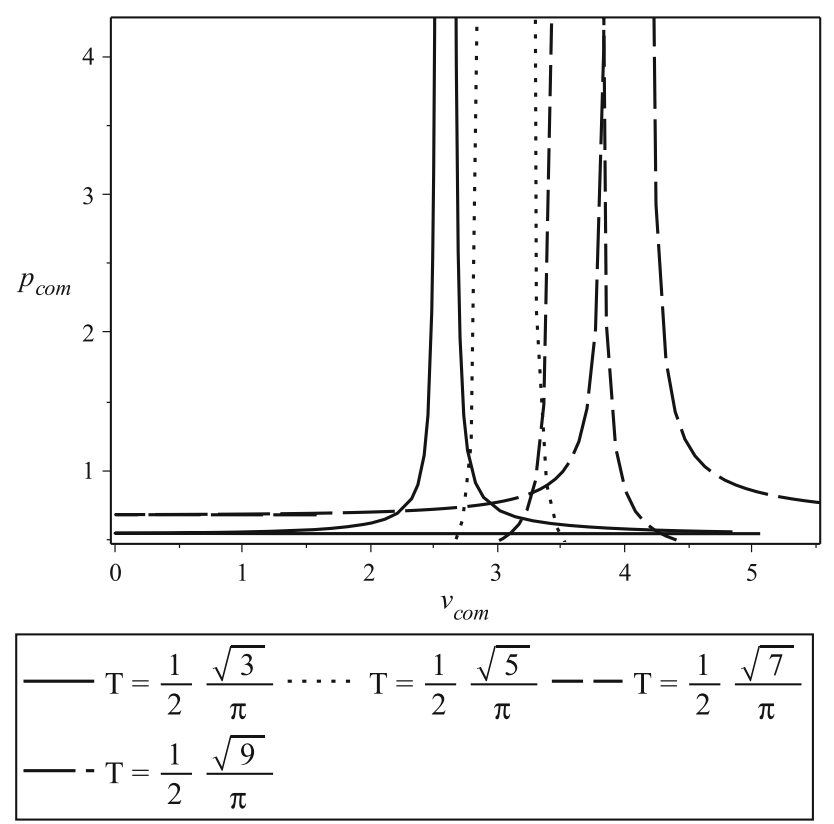

Fig. 13 A $P-V$ diagram between holographic complexity and pressure, showing a totally different behavior from the thermodynamic $P-V$ diagram

break unitarity. However, as the behavior of fidelity susceptibility resembles the behavior of thermodynamics in extended phase space, it can be argued that the thermodynamics of a Reissner-Nordström-AdS black hole is represented by a unitarity process. This can be used as a proposal to resolve the black hole information paradox in a Reissner-NordströmAdS black hole.

\section{SAdS for any dimensions}

In this section we will extend our previous result to higher dimensional AdS spacetimes when there is no electric charge. The line-element of a Schwarzschild-AdS spacetime for any dimension $n$ can be written as follows:

$\mathrm{d} s^{2}=-f(r) \mathrm{d} t^{2}+\frac{\mathrm{d} r^{2}}{f(r)}+r^{2} \mathrm{~d} \Omega_{(n-2)}$,

where $\mathrm{d} \Omega_{n-2}$ denotes the metric of a $S_{n-2}$ sphere defined as

$$
\begin{aligned}
\mathrm{d} \Omega_{n-2}= & \mathrm{d} \theta_{1}^{2}+\frac{1}{k} \sin ^{2}\left(\sqrt{k} \theta_{1}\right) d \theta_{2}^{2} \\
& +\frac{1}{k} \prod_{i=1}^{n-3} \sin ^{2}\left(\sqrt{k} \theta_{i}\right) d \theta_{n-2},
\end{aligned}
$$

where $k=\{-1,0,1\}$ and the function $f(r)$ is

$f(r)=1-\frac{2 M}{r^{n-3}}+\frac{r^{2}}{l^{2}}$.

Clearly, if we set $n=4$ we recover the case studied in Sect. 2 . Now, at the horizon $r_{H}$, the function $f(r)=0$ so that the mass of the black hole and its horizons satisfy the following equation:

$M=r_{H}^{n-3}\left(1+\frac{r_{H}^{2}}{l^{2}}\right)$.

Let us now use the same approach that we used before to compute the area of the minimal surface $\gamma_{A}$. By taking a time slice at $t=0$ in the above line-element, we obtain

$$
\left.\mathrm{d} s^{2}\right|_{t=0}=\left(\frac{\left(\frac{\mathrm{d} r}{\mathrm{~d} \theta}\right)^{2}}{f(r)}+r^{2}\right) \mathrm{d} x^{2}+r^{2}(x) \underbrace{\sum_{a=2}^{n-2}\left(\mathrm{~d} x^{a}\right)^{2}}_{n-3}
$$

where we have parametrized the surface as $r=r(\theta)$ and we have assumed that the $x^{a}$ coordinate lies between $-L / 2 \leq$ $x^{a} \leq L / 2$ where $L$ is the total entangled length of the subsystem on boundary. Hence, the area of the minimal surface can be expressed as follows:

Area $\equiv A=L^{n-3} \int r^{n-3} \sqrt{r^{2}+\frac{r^{\prime 2}}{f(r)}} \mathrm{d} x$

Note that in the above equation, the Lagrangian density $L=L\left(r, r^{\prime}\right)$ does not have the coordinate $x$, i.e. $\frac{\partial L}{\partial x}=0$, so that this term can be written outside the integral. Explicitly, 
this Lagrangian has a conserved charge. If we think on $x$ as time coordinate in dynamical system approach, one needs to satisfy the following constraint:

$r^{\prime} \frac{\partial L}{\partial r^{\prime}}-L=C \equiv$ const,

and therefore we have

$\frac{\partial L}{\partial r^{\prime}}=r^{n-3} \frac{\frac{r^{\prime}}{f(r)}}{\sqrt{r^{2}+\frac{r^{\prime 2}}{f(r)}}}$.

The term in the integrand in Eq. (4.6) can be simplified as

$$
\begin{aligned}
& L^{n-3}\left\{r^{n-3} \frac{\frac{r^{\prime 2}}{f(r)}}{\sqrt{r^{2}+\frac{r^{\prime 2}}{f(r)}}}-r^{n-3} \sqrt{r^{2}+\frac{r^{\prime 2}}{f(r)}}\right\} \\
& =L^{n-3}\left\{r^{n-3}\right\}\left(-\frac{r^{2}}{\sqrt{r^{2}+\frac{r^{\prime 2}}{f(r)}}}\right)=C .
\end{aligned}
$$

The appropriate boundary conditions are given at the turning point $r^{*}$ as $\left.r^{\prime}\right|_{r=r^{*}}=0$ and using this boundary condition in (4.7), we obtain

$$
C=L^{n-3}\left(r_{*}\right)^{n-3}\left(-r_{*}\right)=-L^{n-3}\left(r_{*}\right)^{n-2} .
$$

Using this expression we are able to simplify the integrand in Eq. (4.6) as follows:

$$
\begin{aligned}
A & =2 L^{n-3} \int_{0}^{r^{*}} r^{n-3} x r\left(\frac{r}{r^{*}}\right)^{n-2} \mathrm{~d} x \\
& =2 x \frac{L^{n-3}}{r^{* n-2}} \int_{0}^{r^{*}} r^{2 n-4} \frac{\mathrm{d} x}{\mathrm{~d} r} \mathrm{~d} r \\
& =2 x \frac{L^{n-3}}{r^{* n-2}} \int_{0}^{r^{*}}(r(x))^{2 n-4} \mathrm{~d} x .
\end{aligned}
$$

The corresponding maximal volume is given by the following expression:

$$
\begin{aligned}
V_{\max } & =\int \frac{d r}{\sqrt{f(r)}} r^{n-1} \int \mathrm{d} x L^{n-3} \\
& =L^{n-3} \int_{0}^{r^{*}} \frac{\mathrm{d} r}{\sqrt{f(r)}} r^{n-1} x(r) .
\end{aligned}
$$

Now we consider the case that $n=5$. Five dimensional black objects have widely been studied in the literature because they could have different topologies from the case that $n=4$ [48]. As in the previous sections, we are able to define the fidelity pressure using $V_{\max }$. The complete expression is written in Appendix C. From Figs. 13, 14, and 15 , it may be noted that here again we observe that the behavior of the fidelity susceptibility is similar to the thermodynamics volume. However, the behavior of the holographic complexity is very different from the behavior of both the

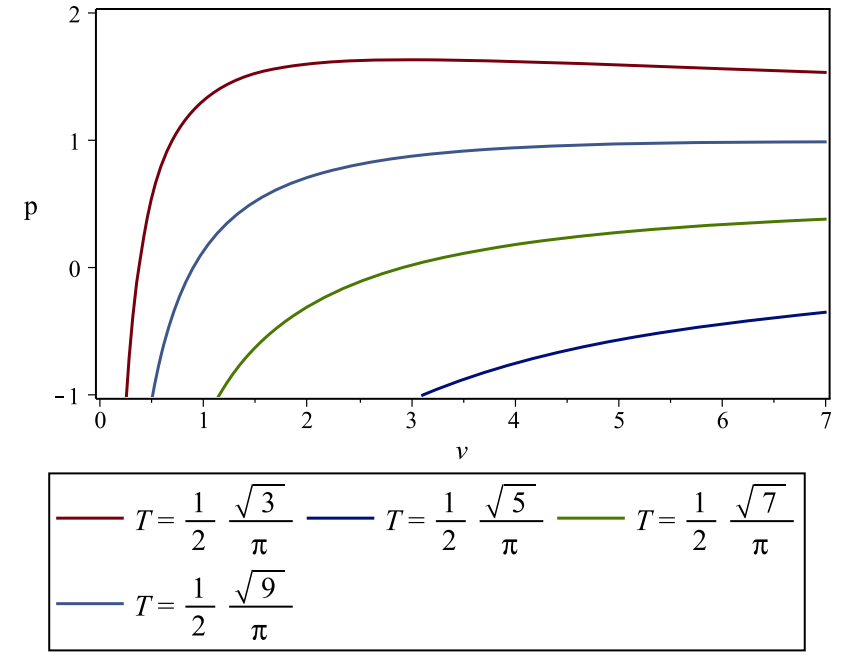

Fig. $14 P-V$ diagrams for the thermodynamic volume and pressure for five dimensional SAdS black holes

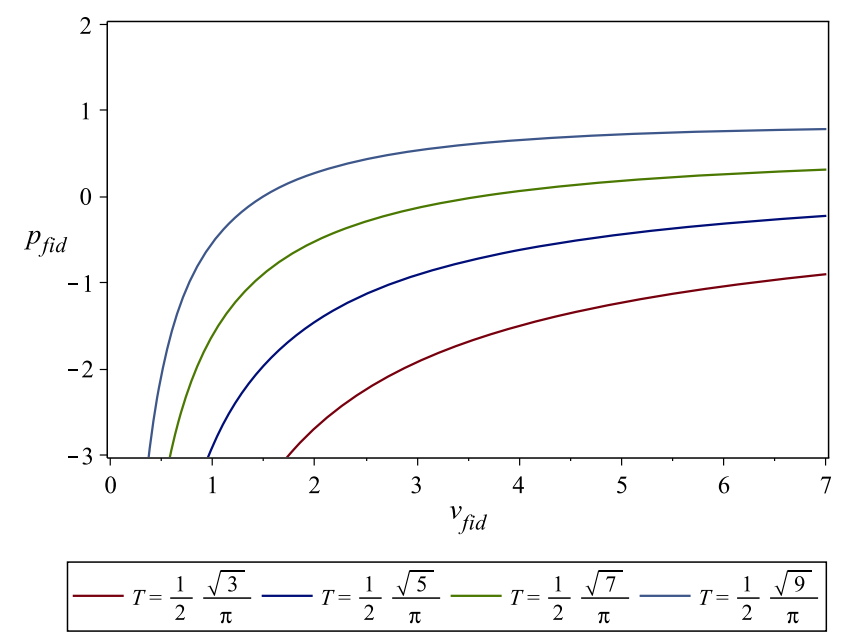

Fig. 15 Five dimensional SAdS black holes showing the behavior of fidelity susceptibility versus pressure. We address the temperatures of this higher dimensional black hole. We indicate that the association of fidelity susceptibility to thermodynamic volume is universal for any number of dimensions

fidelity susceptibility and the thermodynamic volume. This indicates that the behavior is not a property of the specific metric but seems to be a universal behavior of fidelity susceptibility, holographic complexity, and thermodynamic volume. The dual to a higher dimensional Schwarzschild-AdS black hole has also been constructed [46,47]. It is possible to analyze the behavior of fidelity susceptibility of this dual theory, and it this would be described by a unitarity process. As the behavior of a fidelity susceptibility of a higher dimensional Schwarzschild-AdS black hole is similar to the behavior of its thermodynamics in extended phase space, the thermodynamics of a Schwarzschild-AdS black hole is expected to be described by a unitarity process. Furthermore, as this property seems to be of universal and not a property 
of a specific metric, this can be used as a proposal for the black hole information paradox.

\section{Conclusions}

In this paper, we studied the connection between the information dual to different volumes in the bulk of a deformed AdS spacetime and the thermodynamic volume in extended phase space. In the extended phase space, the cosmological constant can be related to the thermodynamic pressure, and a conjugate thermodynamic volume for this pressure can be defined. Furthermore, the information dual to different volumes in the bulk AdS space was measured by the fidelity susceptibility and the holographic complexity. As these quantities diverged, we used a regularized definition for these quantities. We regularized them by subtracting the contribution from the background AdS spacetime from the deformation of the AdS spacetime. It was possible to use this regularized fidelity susceptibility and regularized holographic complexity to define the pressure for these quantities. Thus, we analyzed the $P-V$ equation for these quantities and compared it with the $P-V$ form for the thermodynamic volume and thermodynamic pressure in the extended phase space. It was observed that the regularized fidelity susceptibility showed the same behavior as the thermodynamic volume. The regularized holographic complexity had a very different behavior. We observed this phenomenon for different black holes. This indicates that this is a universal behavior of the fidelity susceptibility, holographic complexity, and thermodynamic volume, and not a property of the specific geometry. It may be noted that fidelity susceptibility of the bulk has a well defined boundary dual. Thus, as the fidelity susceptibility and the thermodynamic volume of the black holes show similar behaviors, it is expected that they represent the same physical quantity. Now it is well known that the evolution of the fidelity susceptibility can be understood in the dual picture from the evolution of a conformal field theory. As the evolution of the conformal field theory is a unitary process, it is expected that the evolution of the fidelity susceptibility in the bulk will also be a unitary process. Now as the fidelity susceptibility can be related to the black hole thermodynamics, it can be argued that the black hole thermodynamics in the extended phase space would be dual to a unitary process. This might help to resolve black hole information paradox.
It would be interesting to generalize this analysis to timedependent geometries. The time-dependent holographic complexity for such time-dependent geometries has been recently studied [49]. It is possible to study the fidelity susceptibility of such time-dependent geometries. It would be interesting to analyze the behavior of $P-V$ diagrams for the holographic complexity and the fidelity susceptibility for such time-dependent geometries. It would also be interesting to analyze the thermodynamics of black holes in such a time-dependent geometry, and compare the thermodynamics of the black hole in extended phase space to fidelity susceptibility and holographic complexity. It is expected that again the fidelity susceptibility and the thermodynamics in extended phase space will have similar behaviors, and the holographic complexity will show a very different behavior.

The black hole thermodynamics has been studied in massive gravity [50]. It would be interesting to obtain the fidelity susceptibility and the holographic complexity for black hole geometries in massive gravity. It is again mentioned that these will diverge, but they can be regularized by subtracting the contribution of background AdS spacetime from the deformation of the AdS spacetime. It would be interesting to analyze the behavior of the $P-V$ diagrams for the fidelity susceptibility and the holographic complexity in massive gravity. This can be compared to the thermodynamics in extended phase space. It is important to understand the behavior of such $P-V$ diagrams for massive gravity, to understand if a similar behavior of fidelity susceptibility and thermodynamics in extended phase space is restricted to Einstein gravity, if it also exists in massive gravity. The complexity was extensively studied for many interesting physical systems like in one-dimensional holographic superconductors [51], charged black holes [52,53], black holes in $f(R)$ gravity [54], singular surfaces [55], AdS black holes [56,57] and massive gravity [58]. It would be interesting to extend our work to these systems.

Acknowledgements The authors thank Sebastian Bahamonde for contributions in an early draft of this work.

Open Access This article is distributed under the terms of the Creative Commons Attribution 4.0 International License (http://creativecomm ons.org/licenses/by/4.0/), which permits unrestricted use, distribution, and reproduction in any medium, provided you give appropriate credit to the original author(s) and the source, provide a link to the Creative Commons license, and indicate if changes were made.

Funded by SCOAP ${ }^{3}$. 


\section{Appendix A: SAdS}

The expressions of volume and pressure are

$$
\begin{aligned}
& V=\frac{1}{2}\left(-\frac{\left(\frac{2}{3} \pi T-\frac{1}{3} \sqrt{4 \pi^{2} T^{2}-3}\right)^{3}}{\sqrt{1+\rho^{2}}}+\frac{\left(\frac{2}{3} \pi T-\frac{1}{3} \sqrt{4 \pi^{2} T^{2}-3}\right)^{3}}{\sqrt{\left(\frac{2}{3} \pi T-\frac{1}{3} \sqrt{4 \pi^{2} T^{2}-3}\right)^{2}+1}}\right) \theta_{0}^{2}, \\
& P=-\frac{a\left(1+3\left(\frac{2}{3} \pi T-\frac{1}{3} \sqrt{4 \pi^{2} T^{2}-3}\right)^{2}\right)\left(\left(\frac{2}{3} \pi T-\frac{1}{3} \sqrt{4 \pi^{2} T^{2}-3}\right)^{2}+1\right)^{3 / 2}}{\left(\frac{2}{3} \pi T-\frac{1}{3} \sqrt{4 \pi^{2} T^{2}-3}\right)^{4}}
\end{aligned}
$$

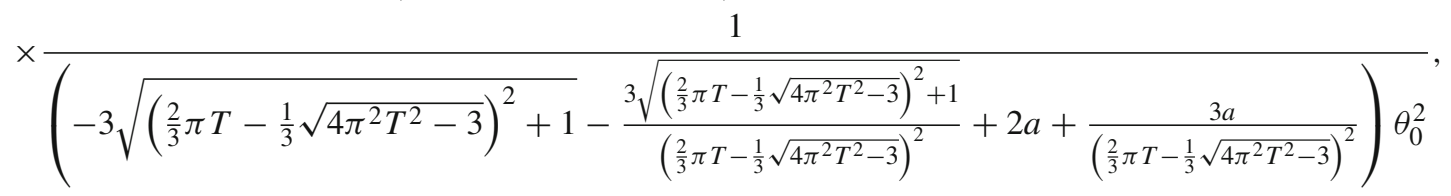

where $a=\sqrt{1+\rho^{2}}$.

\section{Appendix B: RNSAdS}

E-L equation:

$$
\begin{aligned}
\frac{\mathrm{d}^{2} r}{\mathrm{~d} \theta^{2}}= & \frac{1}{-2 r^{2} \sin (\theta) l^{2} \epsilon r_{+}^{3}+2 r \sin (\theta) l^{2} \delta r_{+}^{4}+2 r^{5} \sin (\theta) l^{2}+2 r^{3} \sin (\theta) l^{4}} \\
& \times\left[4 \sin (\theta) r^{8}+8 \sin (\theta) l^{2} r^{6}-8 r^{5} \sin (\theta) \epsilon r_{+}^{3}-2 r^{5} \cos (\theta)\left(\frac{\mathrm{d} r}{\mathrm{~d} \theta}\right) l^{2}+4 r^{4} \sin (\theta) l^{4}\right. \\
& +8 r^{4} \sin (\theta)\left(\frac{\mathrm{d} r}{\mathrm{~d} \theta}\right)^{2} l^{2}+8 r^{4} \sin (\theta) \delta r_{+}^{4}-2 r^{3} \cos (\theta)\left(\frac{\mathrm{d} r}{\mathrm{~d} \theta}\right) l^{4}-8 r^{3} \sin (\theta) l^{2} \epsilon h^{3} \\
& +2 r^{2} \cos (\theta)\left(\frac{\mathrm{d} r}{\mathrm{~d} \theta}\right) l^{2} \epsilon r_{+}^{3}+4 r^{2} \sin (\theta) \epsilon^{2} r_{+}^{6}+6 r^{2} \sin (\theta)\left(\frac{\mathrm{d} r}{\mathrm{~d} \theta}\right)^{2} l^{4}+8 r^{2} \sin (\theta) l^{2} \delta r_{+}^{4} \\
& -5 r \sin (\theta)\left(\frac{\mathrm{d} r}{\mathrm{~d} \theta}\right)^{2} l^{2} \epsilon r_{+}^{3}-2 r(\theta) \cos (\theta)\left(\frac{\mathrm{d} r}{\mathrm{~d} \theta}\right) l^{2} \delta r_{+}^{4}-8 r \sin (\theta) \epsilon r_{+}^{7} \delta \\
& \left.-2 r \cos (\theta)\left(\frac{\mathrm{d} r}{\mathrm{~d} \theta}\right)^{3} l^{4}+4 \sin (\theta) \delta^{2} r_{+}^{8}+4 \sin (\theta)\left(\frac{\mathrm{d} r}{\mathrm{~d} \theta}\right)^{2} l^{2} \delta r_{+}^{4}\right] .
\end{aligned}
$$

The functional $L(\theta)$ with the function $r(\theta)$ given by (3.4) is

$$
\begin{aligned}
L= & -\frac{3}{32 \rho^{2}} \\
& \times\left[-4 \theta^{4} \rho^{7}-\frac{56}{9} \theta^{2}\left(\frac{6}{7}+\theta^{2}\right) \rho^{5}+5 \theta^{4} \rho^{4} \epsilon r_{+}^{3}+\left(\left(-4 \delta r_{+}^{4}-\frac{20}{9}\right) \theta^{4}-\frac{16}{3} \theta^{2}-\frac{32}{3}\right) \rho^{3}\right. \\
& \left.+\frac{29}{9} r_{+}^{3}\left(\theta^{2}+\frac{48}{29}\right) \theta^{2} \epsilon \rho^{2}-r_{+}^{4} \theta^{2}\left(\left(\epsilon^{2} r_{+}^{2}+\frac{20}{9} \delta\right) \theta^{2}+\frac{16}{3} \delta\right) \rho+\theta^{4} \epsilon r_{+}^{7} \delta\right] \\
& \times \sin (\theta) \sqrt{(\rho+c-d)^{2}+\frac{(2 c-4 d)^{2}}{\left(1+(\rho+c-d)^{2}-\frac{\epsilon r_{+}^{3}}{\rho+c-d}+\frac{\delta r_{+}^{4}}{(\rho+c-d)^{2}}\right) \theta^{2}}}
\end{aligned}
$$


where

$$
\begin{aligned}
c= & \frac{1}{2} \frac{\left(-\rho \epsilon r_{+}^{3}+\delta r_{+}^{4}+\rho^{4}+\rho^{2}\right) \theta^{2}}{\rho}, \\
d= & \frac{1}{96 \rho^{2}}\left[\left(-9 \rho \epsilon^{2} r_{+}^{6}+9 \epsilon r_{+}^{7} \delta+45 \rho^{4} \epsilon r_{+}^{3}+29 \rho^{2} \epsilon r_{+}^{3}\right.\right. \\
& \left.\left.-36 \rho^{3} \delta r_{+}^{4}-20 \rho \delta r_{+}^{4}-36 \rho^{7}-56 \rho^{5}-20 \rho^{3}\right) \theta^{4}\right]
\end{aligned}
$$

Hence, $L$ will be

$$
\begin{aligned}
& +2 r_{+} \delta \rho^{3}+4 r_{+} \delta \rho^{5}+2 r_{+}^{5} \delta^{2} \rho-2 \epsilon \rho^{4} \\
& -4 \epsilon \rho^{6}-r_{+}^{4} \delta \rho^{4} \epsilon-3 \epsilon^{2} r_{+}^{7} \delta \rho-2 \epsilon \rho^{8}+2 r_{+}^{5} \delta^{2} \rho^{3} \\
& \left.+2 r_{+} \delta \rho^{7}+3 \epsilon r_{+}^{8} \delta^{2}+2 \rho^{5} \epsilon^{2} r_{+}^{3}\right] r_{+}^{3} \theta^{2} \\
& +\frac{1}{384} \frac{r_{+}^{3} \theta^{4}}{\rho^{4}(1+a)^{2} a^{8}} \times\left[\left(-320 h \delta \rho^{7}+424 \epsilon r_{+}^{4} \delta \rho^{10}\right.\right. \\
& +108 \epsilon \rho^{2} \delta^{2} r_{+}^{8}-606 \epsilon^{2} r_{+}^{7} \delta \rho^{5}-798 \epsilon^{2} r_{+}^{7} \delta \rho^{7} \\
& +516 \epsilon r_{+}^{8} \delta^{2} \rho^{4}+244 r_{+}^{4} \delta \rho^{4} \epsilon+708 \epsilon r_{+}^{8} \delta^{2} \rho^{6} \\
& +396 \epsilon^{3} r_{+}^{10} \delta \rho^{4}+108 \rho^{6} \epsilon^{3} r_{+}^{6}-36 \rho^{8} \epsilon^{3} r_{+}^{6}
\end{aligned}
$$

$$
\begin{aligned}
L= & \frac{1}{240} \frac{\theta\left(240 l^{4} \rho^{4}+320 l^{4} \rho^{4} \theta^{2}+450 \rho^{8} \theta^{4}+360 \theta^{2} \rho^{6} l^{2}+272 l^{4} \rho^{4} \theta^{4}+720 \theta^{4} l^{2} \rho^{6}\right)}{l^{4} \rho^{2}} \\
& +\frac{1}{240} \frac{\theta\left(-360 \theta^{2} \rho^{3} l^{2} r_{+}^{3}-675 \rho^{5} \theta^{4} r_{+}^{3}-495 \theta^{4} \rho^{3} l^{2} r_{+}^{3}\right) \epsilon}{l^{4} \rho^{2}} \\
& +\left(\frac{1}{240} \frac{\theta\left(420 r_{+}^{4} \theta^{4} \rho^{2} l^{2}+360 r_{+}^{4} \theta^{2} \rho^{2} l^{2}+600 \theta^{4} \rho^{4} r_{+}^{4}\right)}{l^{4} \rho^{2}}-\frac{25}{16} \frac{\theta^{5} r_{+}^{7} \epsilon}{l^{4} \rho}\right) \delta .
\end{aligned}
$$

By integrating we find

$$
\begin{aligned}
\int_{0}^{\theta_{0}} L \mathrm{~d} \theta= & -\frac{1}{1440} \frac{\theta_{0}^{2}\left(675 \theta_{0}^{4} \rho^{4} r_{+}^{3}+540 \rho^{2} \theta_{0}^{2} r_{+}^{3}+375 \theta_{0}^{4} r_{+}^{7} \delta+495 \theta_{0}^{4} \rho^{2} r_{+}^{3}\right) \epsilon}{\rho} \\
& +\frac{1}{1440} \frac{\theta_{0}^{2}\left(450 \theta_{0}^{4} \rho^{7}+272 \theta_{0}^{4} \rho^{3} l^{4}+720 \theta_{0}^{4} \rho^{5}+540 \rho^{5} \theta_{0}^{2}+480 \rho^{3} \theta_{0}^{2}+420 \theta_{0}^{4} \rho \delta r_{+}^{4}\right)}{\rho} \\
& +\frac{1}{1440} \frac{\theta_{0}^{2}\left(+600 \theta_{0}^{4} \rho^{3} \delta r_{+}^{4}+720 \rho^{3}+540 \rho \theta_{0}^{2} \delta r_{+}^{4}\right)}{\rho} .
\end{aligned}
$$

Solving the definite integral (3.9), and leaving only the terms in $\theta$ up to order six yields

$$
\begin{aligned}
V_{1}= & \frac{r_{+}^{3}}{4 a^{3} b^{3}} \times\left[2 a \epsilon \rho^{2}+2 a r_{+}^{2} \delta+2 a r_{+}^{4} \delta+2 a r_{+}^{2} \epsilon \epsilon\right. \\
& -2 b \epsilon \rho^{2}-2 b r_{+}^{2}+2 a \epsilon+4 a r_{+}^{4} \delta \epsilon \rho^{2}+3 a \epsilon r_{+}^{6} \delta \rho^{2} \\
& +2 a r_{+}^{2} \epsilon \rho^{2}+2 a h^{2} \delta \rho^{2}+2 a \rho^{2} \delta r_{+}^{4}+4 a r_{+}^{4} \delta \epsilon \\
& +3 a \epsilon r_{+}^{6} \delta-3 b r_{+}^{4} \delta \epsilon \rho^{2}-3 b r_{+}^{6} \delta \epsilon \rho^{2}-2 b r_{+} \delta \rho \\
& -2 b r_{+}^{3} \delta \rho-2 b r_{+} \delta \rho^{3}-2 b r_{+}^{3} \delta \rho^{3}-4 b r_{+}^{4} \delta \epsilon-4 b r_{+}^{6} \delta \epsilon \\
& -2 b r_{+}^{2} \epsilon \rho^{2}+3 b r_{+}^{4} \delta \epsilon a \rho^{2} \ln \left(\frac{1+a}{\rho}\right) \\
& -3 b r_{+}^{4} \delta \epsilon a \ln \left(\frac{1+b}{r_{+}}\right) \rho^{2}+3 b r_{+}^{6} \delta \epsilon a \rho^{2} \ln \left(\frac{1+a}{\rho}\right) \\
& -3 b r_{+}^{6} \delta \epsilon a \ln \left(\frac{1+b}{r_{+}}\right) \rho^{2}-2 b \epsilon \\
& +3 b h^{6} \delta \epsilon a \ln \left(\frac{1+a}{\rho}\right)+3 b r_{+}^{4} \delta \epsilon a \ln \left(\frac{1+a}{\rho}\right) \\
& \left.-3 b r_{+}^{4} \delta \epsilon a \ln \left(\frac{1+b}{r_{+}}\right)-3 b r_{+}^{6} \delta \epsilon a \ln \left(\frac{1+b}{h}\right)\right] \\
& -\frac{1}{8 a^{5} \rho^{2}} \times\left[-\epsilon r_{+}^{4} \delta \rho^{2}+2 \rho^{3} \epsilon^{2} r_{+}^{3}\right.
\end{aligned}
$$

$$
\begin{aligned}
& -80 r_{+} \delta \rho^{13}-320 r_{+} \delta \rho^{11}-212 \rho^{5} \epsilon^{2} r_{+}^{3} \\
& +288 r_{+}^{9} \delta^{3} \rho^{5}-80 r_{+}^{5} \delta^{2} \rho^{3}-480 r_{+} \delta \rho^{9} \\
& -96 r_{+}^{5} \delta^{2} \rho^{5}-176 \rho^{11} \epsilon^{2} r_{+}^{3}-624 \rho^{7} \epsilon^{2} r_{+}^{3} \\
& +84 \rho^{4} \epsilon^{3} r_{+}^{6}-600 \rho^{9} \epsilon^{2} r_{+}^{3}+378 \epsilon^{3} r_{+}^{10} \delta \rho^{6} \\
& -810 \epsilon^{2} r_{+}^{11} \delta^{2} \rho^{5}+300 \epsilon r_{+}^{8} \delta^{2} \rho^{8}+560 \epsilon \rho^{8} \\
& +432 r_{+}^{12} \delta^{3} \epsilon \rho^{4}+912 \epsilon r_{+}^{4} \delta \rho^{8}+792 \epsilon r_{+}^{4} \delta \rho^{6} \\
& -900 \epsilon^{2} r_{+}^{11} \delta^{2} \rho^{3}+504 r_{+}^{12} \delta^{3} \epsilon \rho^{2}-138 \rho^{3} \epsilon^{2} r_{+}^{7} \delta \\
& -90 \epsilon^{2} r_{+}^{11} \delta^{2} \rho+18 \epsilon^{3} r_{+}^{10} \delta \rho^{2}+352 r_{+}^{5} \delta^{2} \rho^{9} \\
& +192 r_{+}^{5} \delta^{2} \rho^{7}-80 r_{+} \delta \rho^{5}+72 r_{+}^{9} \delta^{3} \rho^{7} a-30 \rho^{10} \epsilon^{3} r_{+}^{6} a \\
& +6 \rho^{13} \epsilon^{2} r_{+}^{3} a+72 r_{+}^{5} \delta^{2} \rho^{11} a \\
& +216 r_{+}^{9} \delta^{3} \rho^{5} a-80 r_{+}^{5} \delta^{2} \rho^{3} a-56 r_{+}^{5} \delta^{2} \rho^{5} a-360 r_{+} \delta \rho^{9} a \\
& +72 r_{+}^{12} \delta^{3} \epsilon a-40 r_{+} \delta \rho^{13} a \\
& -200 r_{+} \delta \rho^{11} a-212 \rho^{5} \epsilon^{2} r_{+}^{3} a-518 \rho^{7} \epsilon^{2} r_{+}^{3} a \\
& -394 \rho^{9} \epsilon^{2} r_{+}^{3} a+84 \rho^{4} \epsilon^{3} r_{+}^{6} a+66 \rho^{6} \epsilon^{3} r_{+}^{6} a \\
& -48 \rho^{8} \epsilon^{3} r_{+}^{6} a-82 \rho^{11} \epsilon^{2} r_{+}^{3} a-80 r_{+} \delta \rho^{5} a \\
& -280 r_{+} \delta \rho^{7} a+200 r_{+}^{5} \delta^{2} \rho^{7} a+248 r_{+}^{5} \delta^{2} \rho^{9} a \\
& +144 r_{+}^{9} \delta^{3} \rho^{3} a+144 r_{+}^{9} \delta^{3} \rho^{3}+60 \epsilon r_{+}^{4} \delta \rho^{12} \\
& +10
\end{aligned}
$$




$$
\begin{aligned}
& +128 \epsilon \rho^{6}+24 \epsilon \rho^{16} a+536 \epsilon \rho^{12} a+184 \epsilon \rho^{14} a \\
& +128 \epsilon \rho^{6} a+496 \epsilon \rho^{8} a+744 \epsilon \rho^{10} a \\
& +72 r_{+}^{1} 2 \delta^{3} \epsilon+144 r_{+}^{9} \delta^{3} \rho^{7}-60 \rho^{10} \epsilon^{3} r_{+}^{6}+12 \rho^{13} \epsilon^{2} r_{+}^{3} \\
& +144 r_{+}^{5} \delta^{2} \rho^{11}+242 \epsilon r_{+}^{4} \delta \rho^{10} a \\
& +108 \epsilon \rho^{2} \delta^{2} r_{+}^{8} a-537 \epsilon^{2} r_{+}^{7} \delta \rho^{5} a-564 \epsilon^{2} r_{+}^{7} \delta \rho^{7} a \\
& +462 \epsilon r_{+}^{8} \delta^{2} \rho^{4} a \\
& +504 \epsilon r_{+}^{8} \delta^{2} \rho^{6} a+387 \epsilon^{3} r_{+}^{10} \delta \rho^{4} a \\
& +468 r_{+}^{12} \delta^{3} \epsilon \rho^{2} a-138 \rho^{3} \epsilon^{2} r_{+}^{7} \delta a \\
& +670 \epsilon r_{+}^{4} \delta \rho^{6} a+244 \epsilon r_{+}^{4} \delta \rho^{4} a \\
& -855 \epsilon^{2} r_{+}^{11} \delta^{2} \rho^{3} a+638 \epsilon r_{+}^{4} \delta \rho^{8} a \\
& +18 \epsilon^{3} r_{+}^{10} \delta a \rho^{2}-90 \epsilon^{2} r_{+}^{11} \delta^{2} a \rho-165 \epsilon^{2} r_{+}^{7} \delta \rho^{9} a \\
& +150 \epsilon r_{+}^{8} \delta^{2} \rho^{8} a+189 \epsilon^{3} r_{+}^{10} \delta \rho^{6} a+216 r_{+}^{12} \delta^{3} \epsilon \rho^{4} a \\
& -405 \epsilon^{2} r_{+}^{11} \delta^{2} \rho^{5} a+30 \epsilon r_{+}^{4} \delta \rho^{12} a+320 \epsilon \rho^{14} \\
& \left.\left.+960 \epsilon \rho^{10}+48 \epsilon \rho^{16}+800 \epsilon \rho^{12}-330 \epsilon^{2} r_{+}^{7} \delta \rho^{9}\right) r_{+}^{3} \theta^{4}\right]
\end{aligned}
$$

Now, by taking series in $\theta$ (up to order four), and leaving only linear terms in $\epsilon$ and $\delta$, we find

$$
\begin{aligned}
& V_{4}=\frac{1}{24 a^{3} b^{3}} \\
& \times\left(-3 \theta^{2} r_{+}^{6} \delta \epsilon b a \ln (1+a) \rho^{2}-3 \theta^{2} r_{+}^{4}\right. \\
& \times \delta \epsilon b a \ln (1+a) \rho^{2}+3 \theta^{2} r_{+}^{6} \delta \epsilon b a \ln (1+b) \rho^{2} \\
& +3 \theta^{2} r_{+}^{6} \delta \epsilon b a \ln (\rho) \rho^{2} \\
& -3 \theta^{2} r_{+}^{4} \delta \epsilon b a \ln \left(r_{+}\right) \rho^{2}+3 \theta^{2} r_{+}^{4} \delta \epsilon b a \ln (1+b) \rho^{2} \\
& -3 \theta^{2} r_{+}^{6} \delta \epsilon b a \ln \left(r_{+}\right) \rho^{2}+3 \theta^{2} r_{+}^{4} \delta \epsilon b a \ln (\rho) \rho^{2} \\
& +12 \epsilon a-12 \epsilon b-18 r_{+}^{4} \delta \epsilon b a \rho^{2} \ln (1+b) \\
& +18 r_{+}^{4} \delta \epsilon b a \rho^{2} \ln \left(r_{+}\right) \\
& -18 r_{+}^{6} \delta \epsilon b a \rho^{2} \ln (1+b)+18 r_{+}^{6} \delta \epsilon b a \rho^{2} \ln \left(r_{+}\right) \\
& +18 r_{+}^{6} \delta \epsilon b a \rho^{2} \ln (1+a)-3 \theta^{2} r_{+}^{6} \delta \epsilon b a \ln (1+a) \\
& -3 \theta^{2} r_{+}^{4} \delta \epsilon b a \ln (1+a) \\
& +3 \theta^{2} r_{+}^{6} \delta \epsilon b a \ln (1+b) \\
& +3 \theta^{2} r_{+}^{6} \delta \epsilon b a \ln (\rho)-3 \theta^{2} r_{+}^{4} \delta \epsilon b a \ln \left(r_{+}\right) \\
& +3 \theta^{2} r_{+}^{4} \delta \epsilon b a \ln (1+b)-3 \theta^{2} r_{+}^{6} \delta \epsilon b a \ln \left(r_{+}\right) \\
& +3 \theta^{2} r_{+}^{4} \delta \epsilon b a \ln (\rho)-18 r_{+}^{6} \delta \epsilon b a \rho^{2} \ln (\rho) \\
& +18 r_{+}^{4} \delta \epsilon b a \rho^{2} \ln (1+a)-18 r_{+}^{4} \delta \epsilon b a \rho^{2} \ln (\rho) \\
& +18 r_{+}^{4} \delta \epsilon b a \ln \left(r_{+}\right)-18 r_{+}^{6} \delta \epsilon b a \ln (1+b) \\
& +18 r_{+}^{6} \delta \epsilon b a \ln \left(r_{+}\right)+18 r_{+}^{4} \delta \epsilon b a \ln (1+a) \\
& -18 r_{+}^{4} \delta \epsilon b a \ln (\rho)+18 r_{+}^{6} \delta \epsilon b a \ln (1+a) \\
& -18 r_{+}^{6} \delta \epsilon b a \ln (\rho)+2 \theta^{2} r_{+}^{3} \delta b \rho^{3}+4 \theta^{2} r_{+}^{6} \delta \epsilon b \\
& +3 \theta^{2} r_{+}^{6} \delta \epsilon b \rho^{2}+4 \theta^{2} r_{+}^{4} \delta \epsilon b+3 \theta^{2} r_{+}^{4} \delta \epsilon b \rho^{2} \\
& -4 \theta^{2} r_{+}^{4} \delta \epsilon a-4 \theta^{2} r_{+}^{4} \delta \epsilon a \rho^{2}-3 \theta^{2} r_{+}^{6} \delta \epsilon a \\
& -3 \theta^{2} r_{+}^{6} \delta \epsilon a \rho^{2}-2 \theta^{2} \epsilon a r_{+}^{2} \rho^{2}+2 \theta^{2} \epsilon b r_{+}^{2} \rho^{2} \\
& -2 \theta^{2} r_{+}^{2} \delta a \rho^{2}-2 \theta^{2} r_{+}^{4} \delta a \rho^{2}
\end{aligned}
$$

$$
\begin{aligned}
& +2 \theta^{2} r_{+} \delta b \rho+2 \theta^{2} r_{+}^{3} \delta b \rho-18 r_{+}^{4} \delta \epsilon b a \ln (1+b) \\
& +6 \theta^{2} \rho^{4} \epsilon b+6 \theta^{2} \rho^{2} \epsilon b-6 \theta^{2} r_{+}^{3} \delta b \rho-6 \theta^{2} r_{+} \delta b \rho^{3} \\
& -6 \theta^{2} r_{+}^{3} \delta b \rho^{3}+6 \theta^{2} \rho^{2} \epsilon b r_{+}^{2}+6 \theta^{2} \rho^{4} \epsilon b r_{+}^{2} \\
& +3 \theta^{2} r_{+}^{4} \delta b \epsilon+3 \theta^{2} r_{+}^{6} \delta b \epsilon-6 \theta^{2} r_{+} \delta b \rho \\
& +2 \theta^{2} r_{+} \delta b \rho^{3}+2 \theta^{2} \epsilon b \rho^{2}+2 \theta^{2} \epsilon b r_{+}^{2}-2 \theta^{2} \epsilon a r_{+}^{2} \\
& -2 \theta^{2} \epsilon a \rho^{2}-2 \theta^{2} r_{+}^{4} \delta a-2 \theta^{2} r_{+}^{2} \delta a-18 r_{+}^{4} \delta \epsilon b \rho^{2} \\
& -18 r_{+}^{6} \delta \epsilon b \rho^{2}+24 r_{+}^{4} \delta \epsilon a \rho^{2}+18 r_{+}^{6} \delta \epsilon a \rho^{2} \\
& -2 \theta^{2} \epsilon a+2 \theta^{2} \epsilon b+12 \epsilon a r_{+}^{2} \rho^{2}-12 \epsilon b r_{+}^{2} \rho^{2} \\
& +12 r_{+}^{2} \delta a \rho^{2}+12 r_{+}^{4} \delta a \rho^{2}-12 r_{+} \delta b \rho-12 r_{+}^{3} \delta b \rho \\
& -12 r_{+} \delta b \rho^{3}-12 r_{+}^{3} \delta b \rho^{3}-24 r_{+}^{6} \delta \epsilon b-24 r_{+}^{4} \delta \epsilon b \\
& +24 r_{+}^{4} \delta \epsilon a+18 r_{+}^{6} \delta \epsilon a-12 \epsilon b \rho^{2} \\
& -12 \epsilon b r_{+}^{2}+12 \epsilon a r_{+}^{2} \\
& \left.+12 \epsilon a \rho^{2}+12 r_{+}^{4} \delta a+12 r_{+}^{2} \delta a\right) r_{+}^{3} \theta,
\end{aligned}
$$

where we have defined $V_{4}=V_{3} \sin (\theta)$. Using these expansions, the complexity volume becomes

$$
\begin{aligned}
& V_{c}=2 \pi \int_{0}^{\theta_{0}} V 4 d \theta \\
& =\frac{1}{48 a^{3} b^{3}} \times\left(6 \theta_{0}^{2} \rho^{4} \epsilon b+6 \theta_{0}^{2} \rho^{2} \epsilon b\right. \\
& +3 \theta_{0}^{2} r_{+}^{4} \delta \epsilon b a \rho^{2} \ln (1+b) \\
& +3 \theta_{0}^{2} r_{+}^{4} \delta \epsilon b a \rho^{2} \ln (\rho) \\
& +3 \theta_{0}^{2} r_{+}^{6} \delta \epsilon b a \rho^{2} \ln (\rho)-3 \theta_{0}^{2} r_{+}^{4} \delta \epsilon b a \rho^{2} \ln (1+a) \\
& +3 \theta_{0}^{2} r_{+}^{6} \delta \epsilon b a \rho^{2} \ln (1+b) \\
& -3 \theta_{0}^{2} r_{+}^{6} \delta \epsilon b a \rho^{2} \ln \left(r_{+}\right)-3 \theta_{0}^{2} r_{+}^{4} \delta \epsilon b a \rho^{2} \ln \left(r_{+}\right) \\
& -3 \theta_{0}^{2} r_{+}^{6} \delta \epsilon b a \rho^{2} \ln (1+a) \\
& +6 \theta_{0}^{2} \rho^{2} \epsilon b r_{+}^{2}+6 \theta_{0}^{2} \rho^{4} \epsilon b r_{+}^{2}+3 \theta_{0}^{2} r_{+}^{4} \delta b \epsilon \\
& +3 \theta_{0}^{2} r_{+}^{6} \delta b \epsilon-6 \theta_{0}^{2} r_{+} \delta b \rho-6 \theta_{0}^{2} r_{+}^{3} \delta b \rho \\
& -6 \theta_{0}^{2} r_{+} \delta b \rho^{3}-6 \theta_{0}^{2} r_{+}^{3} \delta b \rho^{3} \\
& -3 \theta_{0}^{2} r_{+}^{6} \delta \epsilon b a \ln (1+a)+3 \theta_{0}^{2} r_{+}^{4} \delta \epsilon b a \ln (1+b) \\
& -3 \theta_{0}^{2} r_{+}^{4} \delta \epsilon b a \ln \left(r_{+}\right) \\
& +36 r_{+}^{4} \delta \epsilon b a \rho^{2} \ln \left(r_{+}\right)-36 r_{+}^{6} \delta \epsilon b a \rho^{2} \ln (1+b) \\
& +36 r_{+}^{6} \delta \epsilon b a \rho^{2} \ln \left(r_{+}\right) \\
& +36 r_{+}^{6} \delta \epsilon b a \rho^{2} \ln (1+a)-36 r_{+}^{6} \delta \epsilon b a \rho^{2} \ln (\rho) \\
& +36 r_{+}^{4} \delta \epsilon b a \rho^{2} \ln (1+a) \\
& -36 r_{+}^{4} \delta \epsilon b a \rho^{2} \ln (\rho)-36 r_{+}^{4} \delta \epsilon b a \rho^{2} \ln (1+b) \\
& +24 \epsilon a r_{+}^{2} \rho^{2}-24 \epsilon b r_{+}^{2} \rho^{2} \\
& +24 r_{+}^{2} \delta a \rho^{2}+24 r_{+}^{4} \delta a \rho^{2}-24 r_{+} \delta b \rho \\
& -24 r_{+}^{3} \delta b \rho-24 r_{+} \delta b \rho^{3}-24 r_{+}^{3} \delta b \rho^{3} \\
& -48 r_{+}^{6} \delta \epsilon b \\
& -48 r_{+}^{4} \delta \epsilon b+48 r_{+}^{4} \delta \epsilon a+36 r_{+}^{6} \delta \epsilon a \\
& -2 \theta_{0}^{2} \epsilon a-2 \theta_{0}^{2} \epsilon a \rho^{2}+2 \theta_{0}^{2} \epsilon b \\
& +2 \theta_{0}^{2} \epsilon b \rho^{2}-2 \theta_{0}^{2} \epsilon a r_{+}^{2} \rho^{2}+2 \theta_{0}^{2} \epsilon b r_{+}^{2} \rho^{2}
\end{aligned}
$$




$$
\begin{aligned}
& -2 \theta_{0}^{2} r_{+}^{2} \delta a \rho^{2}-2 \theta_{0}^{2} r_{+}^{4} \delta a \rho^{2} \\
& +2 \theta_{0}^{2} r_{+} \delta b \rho+2 \theta_{0}^{2} r_{+}^{3} \delta b \rho+4 \theta_{0}^{2} r_{+}^{6} \delta \epsilon b+4 \theta_{0}^{2} r_{+}^{4} \delta \epsilon b \\
& -4 \theta_{0}^{2} r_{+}^{4} \delta \epsilon a-3 \theta_{0}^{2} h^{6} \delta \epsilon a \\
& -36 r_{+}^{4} \delta \epsilon b a \ln (1+b) \\
& +36 r_{+}^{4} \delta \epsilon b a \ln \left(r_{+}\right)-36 r_{+}^{6} \delta \epsilon b a \ln (1+b) \\
& +36 r_{+}^{6} \delta \epsilon b a \ln \left(r_{+}\right)+36 r_{+}^{4} \delta \epsilon b a \ln (1+a) \\
& -36 r_{+}^{4} \delta \epsilon b a \ln (\rho) \\
& +36 r_{+}^{6} \delta \epsilon b a \ln (1+a)-36 r_{+}^{6} \delta \epsilon b a \ln (\rho)-24 \epsilon b \rho^{2} \\
& -24 \epsilon b r_{+}^{2}+24 \epsilon a r_{+}^{2} \\
& +24 \epsilon a \rho^{2}+24 r_{+}^{4} \delta a+24 r_{+}^{2} \delta a+24 \epsilon a-24 \epsilon b \\
& -36 r_{+}^{4} \delta \epsilon b \rho^{2}-36 r_{+}^{6} \delta \epsilon b \rho^{2} \\
& +48 r_{+}^{4} \delta \epsilon a \rho^{2}+36 r_{+}^{6} \delta \epsilon a \rho^{2}+2 \theta_{0}^{2} r_{+} \delta b \rho^{3} \\
& +2 \theta_{0}^{2} r_{+}^{3} \delta b \rho^{3}+2 \theta_{0}^{2} \epsilon b r_{+}^{2}-2 \theta_{0}^{2} \epsilon a r_{+}^{2} \\
& -2 \theta_{0}^{2} r_{+}^{4} \delta a-2 \theta_{0}^{2} r_{+}^{2} \delta a+3 \theta_{0}^{2} r_{+}^{4} \delta \epsilon b \rho^{2} \\
& +3 \theta_{0}^{2} r_{+}^{6} \delta \epsilon b \rho^{2}-4 \theta_{0}^{2} r_{+}^{4} \delta \epsilon a \rho^{2} \\
& -3 \theta_{0}^{2} r_{+}^{6} \delta \epsilon a \rho^{2}-3 \theta_{0}^{2} r_{+}^{6} \delta \epsilon b a \ln \left(r_{+}\right) \\
& +3 \theta_{0}^{2} r_{+}^{6} \delta \epsilon b a \ln (\rho) \\
& +3 \theta_{0}^{2} r_{+}^{4} \delta \epsilon b a \ln (\rho) \\
& -3 \theta_{0}^{2} r_{+}^{4} \delta \epsilon b a \ln (1+a) \\
& \left.+3 \theta_{0}^{2} r_{+}^{6} \delta \epsilon b a \ln (1+b) \theta_{0}^{2} r_{+}^{3} \pi\right) \text {. }
\end{aligned}
$$

\section{Appendix C: SAdS for any dimension}

$$
\begin{aligned}
& P_{f i d}=-\frac{\partial M}{\partial V_{\mathrm{Fid}}}=-\frac{\frac{\partial M}{\partial r_{+}}}{\frac{\partial V_{\mathrm{Fid}}}{\partial r_{+}}}=24\left(-r_{+}^{2}-3 r_{+}^{4}+Q^{2}\right) b^{5}(1+b) \\
& \times\left(r _ { + } ^ { 4 } \theta _ { 0 } ^ { 2 } \pi \left(252 r_{+}^{8} \delta \epsilon+372 \delta r_{+}^{4} \epsilon-8 r_{+}^{6} \theta_{0}^{2} \delta-48 r_{+}^{5} \theta_{0}^{2} \delta\right.\right. \\
& +18 \theta_{0}^{2} \epsilon \rho-16 \theta_{0}^{2} r_{+} \delta-48 \theta_{0}^{2} r_{+}^{3} \delta \\
& -16 r_{+}^{7} \theta_{0}^{2} \delta+18 \theta_{0}^{2} \epsilon \rho r_{+}^{6}+216 \delta r_{+}^{4} \\
& -6 \theta_{0}^{2} \epsilon+120 \epsilon r_{+}^{2}+120 r_{+}^{2} \delta+96 r_{+}^{6} \delta-96 r_{+} \delta+48 r_{+}^{4} \epsilon \\
& -288 r_{+}^{5} \delta-96 \delta r_{+}^{7}-288 \delta r_{+}^{3}-21 r_{+}^{8} b \theta_{0}^{2} \delta \epsilon \\
& +21 r_{+}^{10} \theta_{0}^{2} \delta \epsilon \ln \left(\frac{1+b}{r_{+}}\right) \\
& +63 r_{+}^{6} \theta_{0}^{2} \delta \epsilon \ln \left(\frac{1+b}{r_{+}}\right)+63 r_{+}^{8} \theta_{0}^{2} \delta \epsilon \ln \left(\frac{1+b}{r_{+}}\right) \\
& +21 r_{+}^{4} \theta_{0}^{2} \delta \epsilon \ln \left(\frac{1+b}{r_{+}}\right) \\
& -504 r_{+}^{6} \delta \epsilon b \ln \left(\frac{1+b}{r_{+}}\right)-252 \delta r_{+}^{4} \epsilon b \ln \left(\frac{1+b}{r_{+}}\right) \\
& +18 r_{+}^{4} \theta_{0}^{2} \epsilon \rho b-252 r_{+}^{8} \delta \epsilon b \ln \left(\frac{1+b}{r_{+}}\right) \\
& +36 \theta_{0}^{2} \epsilon r_{+}^{2} \rho b-31 r_{+}^{4} \theta_{0}^{2} \delta \epsilon b-49 r_{+}^{6} \theta_{0}^{2} \delta \epsilon b \\
& -6 \theta_{0}^{2} \epsilon b+120 \epsilon b r_{+}^{2}-96 r_{+} \delta b-192 \delta r_{+}^{3} b \\
& -96 r_{+}^{5} b \delta+48 r_{+}^{4} \epsilon b+96 r_{+}^{6} \delta b \\
& +216 \delta r_{+}^{4} b+120 r_{+}^{2} \delta b-756 r_{+}^{6} \delta \epsilon \ln \left(\frac{1+b}{r_{+}}\right) \\
& -252 \delta r_{+}^{4} \epsilon \ln \left(\frac{1+b}{r_{+}}\right)-756 r_{+}^{8} \delta \epsilon \ln \left(\frac{1+b}{r_{+}}\right)
\end{aligned}
$$

$$
\begin{aligned}
& -252 r_{+}^{10} \delta \epsilon \ln \left(\frac{1+b}{r_{+}}\right)-16 \theta_{0}^{2} h \delta b \\
& -32 \theta_{0}^{2} r_{+}^{3} \delta b-10 \theta_{0}^{2} \epsilon b r_{+}^{2}+252 r_{+}^{8} \delta \epsilon b \\
& -8 r_{+}^{6} \theta_{0}^{2} \delta b-4 r_{+}^{4} \theta_{0}^{2} \epsilon b-18 r_{+}^{4} \theta_{0}^{2} \delta b-16 r_{+}^{5} b \theta_{0}^{2} \delta \\
& -10 \theta_{0}^{2} r_{+}^{2} \delta b+18 \theta_{0}^{2} \epsilon b \rho+588 r_{+}^{6} \delta \epsilon b+372 \delta r_{+}^{4} \epsilon b \\
& +72 \epsilon b+72 \epsilon+54 r_{+}^{4} \theta_{0}^{2} \epsilon \rho+54 \theta_{0}^{2} \epsilon r_{+}^{2} \rho \\
& -49 r_{+}^{6} \theta_{0}^{2} \delta \epsilon-21 r_{+}^{8} \theta_{0}^{2} \delta \epsilon \\
& -31 r_{+}^{4} \theta_{0}^{2} \delta \epsilon-10 \theta_{0}^{2} \epsilon r_{+}^{2}-18 r_{+}^{4} \theta_{0}^{2} \delta-4 r_{+}^{4} \theta_{0}^{2} \epsilon-10 \theta_{0}^{2} r_{+}^{2} \delta \\
& +588 r_{+}^{6} \delta \epsilon+21 r_{+}^{4} \theta_{0}^{2} \delta \epsilon b \ln \left(\frac{1+b}{r_{+}}\right) \\
& +42 r_{+}^{6} \theta_{0}^{2} \delta \epsilon b \ln \left(\frac{1+b}{r_{+}}\right) \\
& \left.\left.+21 r_{+}^{8} b \theta_{0}^{2} \delta \epsilon \ln \left(\frac{1+b}{r_{+}}\right)\right)\right)^{-1} .
\end{aligned}
$$

Because we are interested in small deformations of the AdS background, taking series in $\epsilon$ and $\theta$, and leaving only linear terms, we have

$$
\begin{aligned}
& P=12\left(-r_{+}^{2}-3 r_{+}^{4}+Q^{2}\right) b^{5}(1+b)\left(72+120 r_{+}^{2}\right. \\
& +48 r_{+}^{4}+120 b r_{+}^{2}+48 b r_{+}^{4}-10 \theta_{0}^{2} r_{+}^{2} \\
& +18 \theta_{0}^{2} r_{+}^{4} \rho b+54 \theta_{0}^{2} \rho r_{+}^{2}+54 \theta_{0}^{2} r_{+}^{4} \rho \\
& +36 \theta_{0}^{2} \rho r_{+}^{2} b-6 \theta_{0}^{2}-10 \theta_{0}^{2} b r_{+}^{2}-4 \theta_{0}^{2} r_{+}^{4} b+18 \theta_{0}^{2} \rho r_{+}^{6} \\
& \left.-6 \theta_{0}^{2} b+72 b+18 \theta_{0}^{2} b \rho+18 \theta_{0}^{2} \rho-4 \theta_{0}^{2} r_{+}^{4}\right) \epsilon \\
& \times\left(r _ { + } ^ { 5 } \theta _ { 0 } ^ { 2 } \pi \left(48+48 b+9 \theta_{0}^{2} h^{3}+5 \theta_{0}^{2} h+24 r_{+}^{4} \theta_{0}^{2}\right.\right. \\
& +4 r_{+}^{5} \theta_{0}^{2}+8 \theta_{0}^{2} r_{+}^{6}+24 \theta_{0}^{2} r_{+}^{2}+8 \theta_{0}^{2} \\
& -48 r_{+}^{5}+48 r_{+}^{6}+144 r_{+}^{4}-108 r_{+}^{3}+144 r_{+}^{2}-60 r_{+} \\
& +96 b r_{+}^{2}+48 b r_{+}^{4}+8 \theta_{0}^{2} b-108 r_{+}^{3} b \\
& -48 r_{+}^{5} b-60 r_{+} b+16 \theta_{0}^{2} b r_{+}^{2}+8 r_{+}^{4} \theta_{0}^{2} b+5 \theta_{0}^{2} r_{+} b \\
& \left.+9 \theta_{0}^{2} r_{+}^{3} b+4 r_{+}^{5} b \theta_{0}^{2}\right)\left(-48 \theta_{0}^{2} r_{+}^{3}-16 \theta_{0}^{2} r_{+}\right. \\
& -18 r_{+}^{4} \theta_{0}^{2}-48 r_{+}^{5} \theta_{0}^{2} \\
& -8 \theta_{0}^{2} r_{+}^{6}-10 \theta_{0}^{2} r_{+}^{2}-16 r_{+}^{7} \theta_{0}^{2} \\
& -288 r_{+}^{5}-96 r_{+}^{7}+96 r_{+}^{6}+216 r_{+}^{4} \\
& -288 r_{+}^{3}+120 r_{+}^{2}-96 r_{+}+120 b r_{+}^{2}+216 b r_{+}^{4}-192 r_{+}^{3} b \\
& -96 r_{+}^{5} b-96 r_{+} b+96 b r_{+}^{6} \\
& -10 \theta_{0}^{2} b r_{+}^{2}-18 r_{+}^{4} \theta_{0}^{2} b-16 \theta_{0}^{2} r_{+} b \\
& \left.\left.-32 \theta_{0}^{2} r_{+}^{3} b-16 r_{+}^{5} b \theta_{0}^{2}-8 \theta_{0}^{2} r_{+}^{6} b\right) \delta^{2}\right)^{-1} \\
& +\frac{24\left(-r_{+}^{2}-3 r_{+}^{4}+Q^{2}\right) b^{5}(1+b)}{\delta} \\
& \times\left(r _ { + } ^ { 4 } \theta _ { 0 } ^ { 2 } \pi \left(-48 \theta_{0}^{2} r_{+}^{3}-16 \theta_{0}^{2} r_{+}\right.\right. \\
& -18 r_{+}^{4} \theta_{0}^{2}-48 r_{+}^{5} \theta_{0}^{2}-8 \theta_{0}^{2} r_{+}^{6}-10 \theta_{0}^{2} r_{+}^{2}-16 r_{+}^{7} \theta_{0}^{2} \\
& -288 r_{+}^{5}-96 r_{+}^{7}+96 r_{+}^{6}+216 r_{+}^{4}-288 r_{+}^{3}+120 r_{+}^{2}-96 r_{+} \\
& +120 b r_{+}^{2}+216 b r_{+}^{4}-192 r_{+}^{3} b \\
& -96 r_{+}^{5} b-96 r_{+} b+96 b r_{+}^{6}-10 \theta_{0}^{2} b r_{+}^{2} \\
& -18 r_{+}^{4} \theta_{0}^{2} b-16 \theta_{0}^{2} r_{+} b-32 \theta_{0}^{2} r_{+}^{3} b-16 r_{+}^{5} b \theta_{0}^{2} \\
& \left.\left.-8 \theta_{0}^{2} r_{+}^{6} b\right)\right)^{-1} \\
& +12\left(-r_{+}^{2}-3 r_{+}^{4}+Q^{2}\right) b^{5}(1+b)\left(-31 r_{+}^{4} \theta_{0}^{2}-49 \theta_{0}^{2} r_{+}^{6}\right. \\
& -21 r_{+}^{8} \theta_{0}^{2}+588 r_{+}^{6}+252 r_{+}^{8}
\end{aligned}
$$




$$
\begin{aligned}
& +372 r_{+}^{4}-252 r_{+}^{4} \ln \left(\frac{1+b}{r_{+}}\right) \\
& +252 r_{+}^{8} b-756 r_{+}^{6} \ln \left(\frac{1+b}{r_{+}}\right)-756 r_{+}^{8} \ln \left(\frac{1+b}{r_{+}}\right) \\
& -252 r_{+}^{1} 0 \ln \left(\frac{1+b}{r_{+}}\right)+21 r_{+}^{8} b \theta_{0}^{2} \ln \left(\frac{1+b}{r_{+}}\right) \\
& +21 \theta_{0}^{2} r_{+}^{4} b \ln \left(\frac{1+b}{r_{+}}\right)+42 \theta_{0}^{2} r_{+}^{6} b \ln \left(\frac{1+b}{r_{+}}\right) \\
& +63 r_{+}^{6} \theta_{0}^{2} \ln \left(\frac{1+b}{r_{+}}\right) \\
& +63 r_{+}^{8} \theta_{0}^{2} \ln \left(\frac{1+b}{r_{+}}\right)-252 r_{+}^{4} b \ln \left(\frac{1+b}{r_{+}}\right) \\
& -252 r_{+}^{8} b \ln \left(\frac{1+b}{r_{+}}\right) \\
& +21 r_{+}^{1} 0 \theta_{0}^{2} \ln \left(\frac{1+b}{r_{+}}\right)-504 r_{+}^{6} b \ln \left(\frac{1+b}{r_{+}}\right) \\
& +21 r_{+}^{4} \theta_{0}^{2} \ln \left(\frac{1+b}{r_{+}}\right)-21 r_{+}^{8} b \theta_{0}^{2}-31 r_{+}^{4} \theta_{0}^{2} b \\
& \left.-49 \theta_{0}^{2} r_{+}^{6} b+588 b r_{+}^{6}+372 b r_{+}^{4}\right) \epsilon \\
& \times\left(r _ { + } ^ { 5 } \theta _ { 0 } ^ { 2 } \pi \left(48+9 \theta_{0}^{2} r_{+}^{3}+5 \theta_{0}^{2} r_{+}\right.\right. \\
& +24 r_{+}^{4} \theta_{0}^{2}+4 r_{+}^{5} \theta_{0}^{2}+8 \theta_{0}^{2} r_{+}^{6}+24 \theta_{0}^{2} r_{+}^{2}+48 r_{+}^{6}+144 r_{+}^{4} \\
& -108 r_{+}^{3}+8 \theta_{0}^{2}-48 r_{+}^{5}-60 r_{+}+144 r_{+}^{2} \\
& +16 \theta_{0}^{2} b r_{+}^{2}+8 r_{+}^{4} \theta_{0}^{2} b+9 \theta_{0}^{2} r_{+}^{3} b \\
& +5 \theta_{0}^{2} r_{+} b+4 r_{+}^{5} b \theta_{0}^{2}-108 r_{+}^{3} b-48 r_{+}^{5} b-60 r_{+} b+48 b \\
& \left.+96 b r_{+}^{2}+48 b r_{+}^{4}+8 \theta_{0}^{2} b\right)\left(-48 \theta_{0}^{2} r_{+}^{3}-16 \theta_{0}^{2} r_{+}\right. \\
& -18 r_{+}^{4} \theta_{0}^{2}-48 r_{+}^{5} \theta_{0}^{2}-8 \theta_{0}^{2} r_{+}^{6} \\
& -10 \theta_{0}^{2} r_{+}^{2}-16 r_{+}^{7} \theta_{0}^{2}+96 r_{+}^{6}+216 r_{+}^{4}-288 r_{+}^{3}-288 r_{+}^{5} \\
& -96 r_{+}^{7}-96 r_{+}+120 r_{+}^{2}-10 \theta_{0}^{2} b r_{+}^{2} \\
& -18 r_{+}^{4} \theta_{0}^{2} b-32 \theta_{0}^{2} r_{+}^{3} b-16 \theta_{0}^{2} r_{+} b \\
& -16 r_{+}^{5} b \theta_{0}^{2}-8 \theta_{0}^{2} r_{+}^{6} b \\
& -192 r_{+}^{3} b-96 r_{+}^{5} b-96 r_{+} b \\
& \left.\left.+96 b r_{+}^{6}+120 b r_{+}^{2}+216 b r_{+}^{4}\right)\right)^{-1} \text {. }
\end{aligned}
$$

The above expression is the equation for the fidelity susceptibility pressure and volume in our model.

\section{References}

1. K.H. Knuth, AIP Conf. Proc. 1305, 3 (2011)

2. P. Goyal, Information 3, 567 (2012)

3. T. Jacobson, Phys. Rev. Lett. 75, 1260 (1995)

4. R.G. Cai, S.P. Kim, JHEP 0502, 050 (2005)

5. G. 't Hooft, Conf. Proc. C 930308, 284 (1993)

6. L. Susskind, J. Math. Phys. 36, 6377 (1995)

7. J.M. Maldacena, Adv. Theor. Math. Phys. 2, 231 (1998)

8. A. Strominger, C. Vafa, Phys. Lett. B 379, 99 (1996)

9. J.M. Maldacena, JHEP 0304, 021 (2003)

10. D. Momeni, A. Altaibayeva, R. Myrzakulov, Int. J. Geom. Methods. Mod. Phys. 11, 1450091 (2014)

11. S. Ryu, T. Takayanagi, Phys. Rev. Lett. 96, 181602 (2006)

12. V.E. Hubeny, M. Rangamani, T. Takayanagi, JHEP 0707, 062 (2007)

13. F. Barahona, J. Phys. A 15, 3241 (1982)
14. M. Troyer, U.J. Wiese, Phys. Rev. Lett 94, 170201 (2005)

15. D. Momeni, A. Myrzakul, R. Myrzakulov, M. Faizal, Int. J. Mod. Phys. A 33, 1850099 (2018)

16. J. Grunenberg, Phys. Chem. Chem. Phys. 13, 10136 (2011)

17. D. Momeni, M. Faizal, R. Myrzakulov, Int. J. Mod. Phys. D 27, 1850103 (2018)

18. M. Stanowski, Complicity 2, 78 (2011)

19. S.W. Hawking, M.J. Perry, A. Strominger, Phys. Rev. Lett. 116, 231301 (2016)

20. L. Susskind, Fortsch. Phys. 64, 24 (2016)

21. D. Stanford, L. Susskind, Phys. Rev. D 90(12), 126007 (2014)

22. D. Momeni, S.A.H. Mansoori, R. Myrzakulov, Phys. Lett. B 756, 354 (2016)

23. M. Alishahiha, Phys. Rev. D 92, 126009 (2015)

24. M. Miyaji, T. Numasawa, N. Shiba, T. Takayanagi, K. Watanabe, Phy. Rev. Lett 115, 261602 (2015)

25. H.T. Quan, Z. Song, X.F. Liu, P. Zanardi, C.P. Sun, Phys. Rev. Lett. 96, 140604 (2006)

26. P. Zanardi, N. Paunkoviic, Phys. Rev. E 74, 031123 (2006)

27. P. Zanardi, P. Giorda, M. Cozzini, Phys. Rev. Lett. 99, 100603 (2007)

28. D. Momeni, M. Faizal, K. Myrzakulov, R. Myrzakulov, Phys. Lett. B 765, 154 (2017)

29. S. Gunasekaran, R.B. Mann, D. Kubiznak, JHEP 1211, 110 (2012)

30. A. Chamblin, R. Emparan, C. Johnson, R. Myers, Phys. Rev. D 60, 064018 (1999)

31. M. Cvetic, S. Gubser, JHEP 9904, 024 (1999)

32. D. Kubiznak, R.B. Mann, JHEP 1207, 033 (2012)

33. N.S. Mazhari, D. Momeni, S. Bahamonde, M. Faizal, R. Myrzakulov, Phys. Lett. B 766, 94 (2017)

34. D. Sarkar, S. Banerjee, J. Erdmenger, PoS CORFU 2016, 092 (2017)

35. S. Karar, S. Gangopadhyay, Phys. Rev. D 98, 026029 (2018)

36. S.J. Zhang, Nucl. Phys. B 929, 243 (2018)

37. O. Ben-Ami, D. Carmi, JHEP 1611, 129 (2016)

38. P. Roy, T. Sarkar, Phys. Rev. D 96, 026022 (2017)

39. W.C. Gan, F.W. Shu, Phys. Rev. D 96(2), 026008 (2017)

40. A.R. Brown, D.A. Roberts, L. Susskind, B. Swingle, Y. Zhao, Phys. Rev. Lett. 116, 191301 (2016)

41. A.R. Brown, D.A. Roberts, L. Susskind, B. Swingle, Y. Zhao, Phys. Rev. D 93, 086006 (2016)

42. T. Springer, Phys. Rev. D 79, 086003 (2009)

43. G. Camilo, Eur. Phys. J. C 76, 682 (2016)

44. D. Galante, M. Schvellinger, JHEP 1207, 096 (2012)

45. X. Bai, Y.P. Hu, B.H. Lee, Y.L. Zhang, JHEP 1211, 054 (2012)

46. A. Nunez, A.O. Starinets, Phys. Rev. D 67, 124013 (2003)

47. R. Maity, P. Roy, T. Sarkar, Phys. Lett. B 765, 386 (2017)

48. R. Emparan, H.S. Reall, Living Rev. Relat. 11, 6 (2008)

49. D. Momeni, M. Faizal, S. Bahamonde, R. Myrzakulov, Phys. Lett. B 762, 276 (2016)

50. S. Fernando, Phys. Rev. D 94, 124049 (2016)

51. M.K. Zangeneh, Y.C. Ong, B. Wang, Phys. Lett. B 771, 235 (2017)

52. J. Couch, W. Fischler, P.H. Nguyen, JHEP 1703, 119 (2017)

53. Ã. Ãứkcãij, E. AydÄśśner, arXiv:1703.09606 [physics.gen-ph]

54. W.D. Guo, S.W. Wei, Y.Y. Li, Y.X. Liu, Eur. Phys. J. C 77(12), 904 (2017)

55. E. Bakhshaei, A. Mollabashi, A. Shirzad, Eur. Phys. J. C 77(10), 665 (2017)

56. J.X. Mo, G.Q. Li, Nucl. Phys. B 923, 168 (2017)

57. K. Bhattacharya, B.R. Majhi, Phys. Rev. D 95, 104024 (2017)

58. W.J. Pan, Y.C. Huang, Phys. Rev. D 95, 126013 (2017) 